\title{
Fischer carbene catalysis of alkynol cycloisomerization: Application to the synthesis of the altromycin B disaccharide
}

\author{
BonSuk Koo and Frank E. McDonald* \\ Department of Chemistry, Emory University, Atlanta, GA 30322
}

\section{SUPPORTING INFORMATION}

Experimental procedures and characterization data for new compounds (part 1)

General procedures 2

Representative examples of alkynyl alcohol synthesis and cycloisomerizations 3 -11

Preparation of alkynyl alcohol $( \pm)-4 \quad 3$

General procedure for alkynyl alcohol cycloisomerizations 4

Synthesis of glycal $( \pm)-5 \quad 4$

Preparation of alkynyl alcohol ( \pm )-6

Synthesis of glycal $( \pm)-7 \quad 5$

Preparation of alkynyl alcohol $8 \quad 6$

Synthesis of glycal 9

Synthesis of glycal $( \pm)-11 \quad 9$

Synthesis of glycal $( \pm)-13 \quad 9$

Preparation of alkynyl alcohol $14 \quad 9$

Synthesis of glycal $\mathbf{1 5} 10$

Synthesis of glycal $\mathbf{1 7} \quad 11$

Synthesis of glycal 19

Comparisons of non-photochemical procedure vs. photochemical procedures 12 - 16

$\begin{array}{ll}\text { Cycloisomerizations of alkynyl alcohol 20a } & 12\end{array}$

Preparation of alkynyl alcohol 20b 13

Cycloisomerizations of alkynyl alcohol 20b 14

Preparation of alkynyl alcohol 20c 15

Cycloisomerizations of alkynyl alcohol 20c 16

$\begin{array}{ll}\text { Synthesis of the altromycin disaccharide } 33 & 17-33\end{array}$

Enantiomer resolution of $( \pm)-28 \quad 17$

Crystal structure of $(+)-28 \quad 19$

Preparation of glycosylated beta-lactam $29 \quad 25$

Preparation of alkynyl ketone $30 \quad 27$

Preparation of alkynyl alcohol $31 \quad 29$

Synthesis of disaccharide glycal $\mathbf{3 2}$ via alkynyl alcohol cycloisomerization 31

Synthesis of disaccharide glycal 33

${ }^{1} \mathrm{H}$ and ${ }^{13} \mathrm{C}$ NMR spectra of new compounds (part 2) $34-68$ 
General procedures: ${ }^{1} \mathrm{H}$ NMR and ${ }^{13} \mathrm{C}$ NMR spectra were recorded on a Varian INOVA-400 spectrometer $\left(400 \mathrm{MHz}\right.$ for ${ }^{1} \mathrm{H}, 100 \mathrm{MHz}$ for $\left.{ }^{13} \mathrm{C}\right)$, or on an INOVA-600 spectrometer $\left(600 \mathrm{MHz}\right.$ for ${ }^{1} \mathrm{H}, 150 \mathrm{MHz}$ for ${ }^{13} \mathrm{C}$ ). NMR spectra were recorded on solutions in deuterated chloroform $\left(\mathrm{CDCl}_{3}\right)$, with residual chloroform $\left(\delta 7.26 \mathrm{ppm}\right.$ for ${ }^{1} \mathrm{H}$ NMR and $\delta 77.0 \mathrm{ppm}$ for ${ }^{13} \mathrm{C}$ NMR) or deuterated methyl sulfoxide (DMSO- $d_{6}$ ), with residual methyl sulfoxide ( $\delta 2.50 \mathrm{ppm}$ for ${ }^{1} \mathrm{H}$ NMR and $\delta 35.0 \mathrm{ppm}$ for ${ }^{13} \mathrm{C} N M R$ ) taken as the standard, and were reported in parts per million (ppm). Abbreviations for signal coupling are as follows: s, singlet; $d$, doublet; $t$, triplet; q, quartet; $m$, multiplet. IR spectra were collected on a Mattson Genesis II FT-IR spectrometer as neat films. Mass spectra (high resolution FAB or El) were recorded on a VG 70-S Nier Johason Mass spectrometer or a Thermo Finnigan LTQ FT spectrometer. Melting points were determined with a Fisher-Johns melting point apparatus. Optical rotations were measured at $23^{\circ} \mathrm{C}$ (concentration in $\mathrm{g} / 100 \mathrm{~mL}$ ) using a Perkin-Elmer 341 polarimeter. Analytical Thin Layer Chromatography (TLC) was performed on precoated glass backed plates purchased from Whatman (silica gel $60 \mathrm{~F}_{254} ; 0.25 \mathrm{~mm}$ thickness). Flash column chromatography was carried out with silica gel 60 (230-400 mesh ASTM) from EM Science.

All reactions were carried out with anhydrous solvents in oven-dried or flamedried and nitrogen- or argon-charged glassware. Anhydrous solvents except as mentioned were dried with 3 or $4 \AA$ molecular sieves (beads) purchased from Aldrich and tested for trace water content with Coulometric KF Titrator from Denver Instruments.

All solvents used in extraction procedures and chromatography were used as received from commercial suppliers without prior purification. During reaction workup, the reaction mixture was usually diluted to three times the original volume, and washed with an equal volume of water and/or aqueous solutions as needed. All reagents were purchased from Aldrich or Strem Chemicals. 


\section{Representative examples of alkynyl alcohol synthesis and cycloisomerizations}

\section{Preparation of alkynyl alcohol ( \pm )-4}

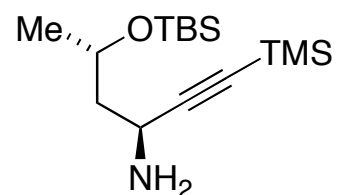

$( \pm)-\mathbf{A}$

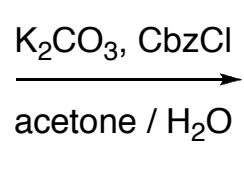

acetone $/ \mathrm{H}_{2} \mathrm{O}$

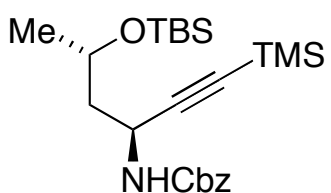

$( \pm)-B$

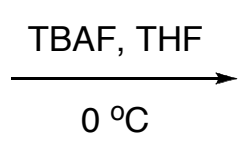

$( \pm)-4$

Cbz protection: The known amine $\mathbf{A}^{1}(0.380 \mathrm{~g}, 1.26 \mathrm{mmol})$ was dissolved in a mixture of acetone $(3 \mathrm{~mL})$ and water $(3 \mathrm{~mL}), \mathrm{K}_{2} \mathrm{CO}_{3}(17.4 \mathrm{mg}, 0.126 \mathrm{mmol})$ and $\mathrm{CbzCl}(0.266$ $\mathrm{mL}, 1.89 \mathrm{mmol}$ ) were added quickly. The mixture was stirred at room temperature for 10 min, and then water $(3 \mathrm{~mL})$ was added and the reaction mixture diluted with $\mathrm{CH}_{2} \mathrm{Cl}_{2}$ (3 $\mathrm{mL}$ ). The organic layer was separated and the aqueous layer was extracted with $\mathrm{CH}_{2} \mathrm{Cl}_{2}$ $(3 \times 5 \mathrm{~mL})$. The combined organic layer was dried over $\mathrm{MgSO}_{4}$ and evaporated under reduced pressure. Chromatography (hexanes : EtOAc $=15: 1$ ) gave $\mathrm{Cbz}$ protected compound B as a white crystal $(0.367 \mathrm{~g}, 81 \%$ yield $) . \quad \mathrm{MP}=100-101^{\circ} \mathrm{C} ;{ }^{1} \mathrm{H}$ NMR $(600$ $\mathrm{MHz}, \mathrm{CDCl}_{3}$ ) $\delta$ 7.36-7.11 (m, 5H), 5.11 (s, 2H), 4.96 (brs, 1H), 4.64 (brs, 1H), 4.01 (brs, $1 \mathrm{H}$ ), 1.85 (brs, 1H), 1.60 (ddd, $J=3.0,10.8 \mathrm{~Hz}, 1 \mathrm{H}$ ), 1.18 (d, J = 6.6 Hz, 3H), 0.84 (s, $9 \mathrm{H}), 0.08$ (s, 9H), 0.02 (s, 6H); ${ }^{13} \mathrm{C}$ NMR (150 MHz, $\left.\mathrm{CDCl}_{3}\right) \delta 155.31,136.61,128.75$, 128.37, 105.16, 88.45, 67.06, 66.23, 46.15, 42.26, 26.11, 24.38, 18.24, 0.14, 0.09, -3.92, -4.67 ; IR (KBr) 3286, 2958, 2930, 2895, 2857, 2171, 1693, 1538, 1251, $840 \mathrm{~cm}^{-1}$; HRMS [M+H] Calcd. for $\mathrm{C}_{23} \mathrm{H}_{40} \mathrm{O}_{3} \mathrm{~N}_{1}{ }^{28} \mathrm{Si}_{2}, 434.25413$, Found. 434.25389.

Desilylation: Compound B $(0.367 \mathrm{~g}, 1.02 \mathrm{mmol})$ was dissolved in THF $(6 \mathrm{~mL})$, cooled to $0{ }^{\circ} \mathrm{C}$, and TBAF $(4.10 \mathrm{~mL}, 1 \mathrm{M}$ in THF) was added and then stirred for $1 \mathrm{~h}$. The reaction was quenched with water and diluted with EtOAc $(5 \mathrm{~mL})$, and then brine $(5 \mathrm{~mL})$ was added. The organic layer was separated and the aqueous layer was extracted with EtOAc $(3 \times 10 \mathrm{~mL})$. The combined organic layer was dried over $\mathrm{MgSO}_{4}$ and evaporated under reduced pressure. Chromatography (hexanes : EtOAc $=2: 1$ to $1: 1$ ) gave the alkynyl alcohol $( \pm)-4$ as a colorless oil $\left(0.229 \mathrm{~g}, 91 \%\right.$ yield). ${ }^{1} \mathrm{H} \mathrm{NMR}\left(400 \mathrm{MHz}, \mathrm{CDCl}_{3}\right)$ $\delta 7.36-7.30(\mathrm{~m}, 5 \mathrm{H}), 5.19$ (brs, 1H), $5.11(\mathrm{~s}, 2 \mathrm{H}), 4.66(\mathrm{~d}, J=7.2 \mathrm{~Hz}, 1 \mathrm{H}), 4.03$ (dqd, $J=$

(1) Davidson, M. H.; McDonald, F. E. Org. Lett. 2004, 6, 1601. 
3.6, 6.0, 10.0 Hz, 1H), 2.34 (d, J = 2.0 Hz, 1H), 2.05 (brs, 1H), 1.89-1.76 (m, 2H), 1.24 $(\mathrm{d}, J=6.0 \mathrm{~Hz}, 3 \mathrm{H}) ;{ }^{13} \mathrm{C}$ NMR $\left(100 \mathrm{MHz}, \mathrm{CDCl}_{3}\right) \delta 155.65,136.36,128.76,128.44$, 88.14, 72.12, 67.29, 65.57, 44.94, 41.53, 24.12; IR (neat) 3402 (brs), 2965, 2925, 1689, 1530, $1249 \mathrm{~cm}^{-1}$; HRMS Calcd [M+H]. for $\mathrm{C}_{14} \mathrm{H}_{18} \mathrm{O}_{3} \mathrm{~N}_{1}, 248.12812$, Found. 248.12800.

\section{General procedure for alkynyl alcohol cycloisomerizations:}

The alkynyl alcohol $(0.1 \mathrm{mmol})$ was dissolved in dry THF $(1.0 \mathrm{~mL})$ in a $5 \mathrm{~mL}$ conical vial, and then $\mathrm{Et}_{3} \mathrm{~N}(0.14 \mathrm{~mL}, 1.0 \mathrm{mmol})$ and tungsten carbene $3(9.6 \mathrm{mg}, 0.025 \mathrm{mmol})$ were added under argon atmosphere. The vial was sealed with a Teflon cap, and then stirred for $12 \mathrm{~h}$ at $40^{\circ} \mathrm{C}$, after which time the reaction mixture was cooled to room temperature. Solvent was removed by rotary evaporation. The yellowish crude oil was purified by chromatography (hexanes : $E t O A c=20: 1$ with $1 \% \mathrm{Et}_{3} \mathrm{~N}$ ) to give pure product glycal.

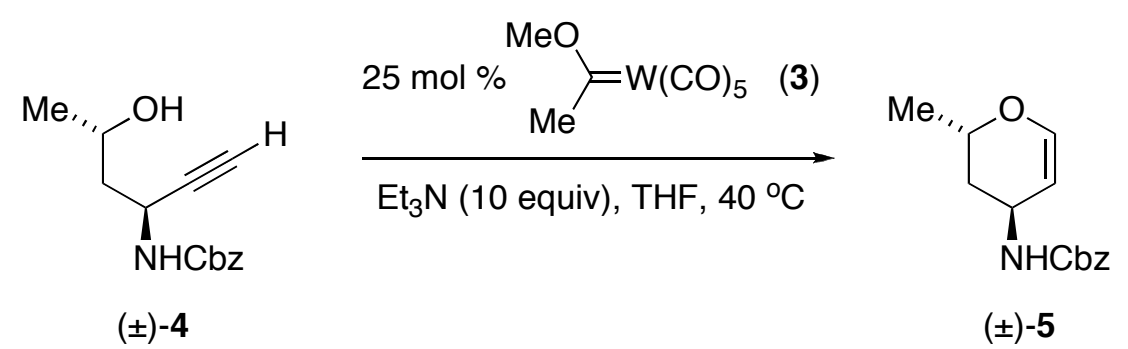

Synthesis of glycal ( $\mathbf{\pm})-5$ : Following the general procedure for alkynyl alcohol cycloisomerization, alkynyl alcohol $( \pm)-4(25 \mathrm{mg}, 0.10 \mathrm{mmol})$ afforded glycal $( \pm)-5$ as a white crystal $\left(23 \mathrm{mg}, 92 \%\right.$ yield). MP $=92-93{ }^{\circ} \mathrm{C} ;{ }^{1} \mathrm{H}$ NMR $\left(400 \mathrm{MHz}, \mathrm{CDCl}_{3}\right) \delta 7.37-$ $7.30(\mathrm{~m}, 5 \mathrm{H}), 6.49(\mathrm{~d}, J=6.4 \mathrm{~Hz}, 1 \mathrm{H}), 5.13(\mathrm{~d}, J=12.4 \mathrm{~Hz}, 1 \mathrm{H}), 5.06(\mathrm{~d}, J=12.4 \mathrm{~Hz}$, $1 \mathrm{H}), 4.83(\mathrm{brs}, 1 \mathrm{H}), 4.75$ (dt, $J=1.6,6.0 \mathrm{~Hz}, 1 \mathrm{H}), 4.14(\mathrm{~d}, J=6.0 \mathrm{~Hz}, 1 \mathrm{H}), 3.86(\mathrm{dqd}, J$ $=1.6,6.0,12.4 \mathrm{~Hz}, 1 \mathrm{H}), 1.95(\mathrm{~d}, J=14.4 \mathrm{~Hz}, 1 \mathrm{H}$ ), 1.67 (ddd, $J=4.4,12.4 \mathrm{~Hz}, 1 \mathrm{H}), 1.31$ $(\mathrm{d}, J=6.0 \mathrm{~Hz}, 3 \mathrm{H}) ;{ }^{13} \mathrm{C}$ NMR $\left(100 \mathrm{MHz}, \mathrm{CDCl}_{3}\right) \delta 155.24,147.69,136.61,128.78$, 128.42, 99.75, 68.19, 66.89, 41.82, 36.84, 20.98; IR (KBr) 1680, 1528, $12391070 \mathrm{~cm}^{-1}$; HRMS [M+H] Calcd. for $\mathrm{C}_{14} \mathrm{H}_{18} \mathrm{O}_{3} \mathrm{~N}_{1}, 248.12812$, Found. 248.12793. 


\section{Preparation of alkynyl alcohol ( \pm )-6}

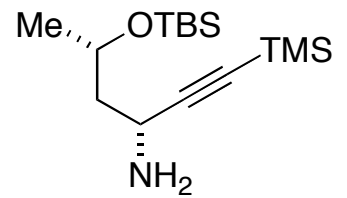

$( \pm)-\mathbf{C}$

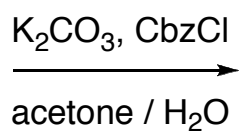

acetone $/ \mathrm{H}_{2} \mathrm{O}$

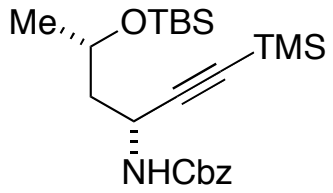

$( \pm)-\mathbf{D}$

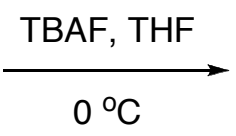

Cbz protection: The procedure as described above for $( \pm)-4$ was followed with the known amine $\mathbf{C}^{1}(0.210 \mathrm{~g}, 0.701 \mathrm{mmol})$ and $\mathrm{CbzCl}(0.148 \mathrm{~mL}, 1.05 \mathrm{mmol})$, giving Cbzprotected compound D as a colorless oil $\left(0.230 \mathrm{~g}, 75 \%\right.$ yield). ${ }^{1} \mathrm{H}$ NMR $(400 \mathrm{MHz}$, $\left.\mathrm{CDCl}_{3}\right) \delta 7.35-7.28(\mathrm{~m}, 5 \mathrm{H}), 5.81(\mathrm{~d}, J=8.4 \mathrm{~Hz}, 1 \mathrm{H}), 5.11(\mathrm{~s}, 2 \mathrm{H}), 4.58$ (brs, 1H), 4.25 (brs, 1H), 1.82-1.73 (m, 2H), $1.18(\mathrm{~d}, J=6.0 \mathrm{~Hz}, 3 \mathrm{H}), 0.90(\mathrm{~s}, 9 \mathrm{H}), 0.15(\mathrm{~s}, 9 \mathrm{H}), 0.12(\mathrm{~s}$, $3 \mathrm{H}), 0.09$ (s, 3H); ${ }^{13} \mathrm{C} \mathrm{NMR}\left(100 \mathrm{MHz}, \mathrm{CDCl}_{3}\right) \delta 155.54,136.87,128.62,128.13,128.04$, 104.91, 87.84, 66.92, 66.80, 43.77, 42.40, 26.12, 24.19, 18.19, 0.15, -3.88, -4.75; IR (neat) 3289, 2957, 2930, 2171, 1729, 1500, 1251, $841 \mathrm{~cm}^{-1}$; HRMS [M+H] Calcd. for $\mathrm{C}_{23} \mathrm{H}_{40} \mathrm{O}_{3} \mathrm{~N}_{1}{ }^{28} \mathrm{Si}_{2}, 434.25413$, Found. 434.25432.

Desilylation: The procedure as described above for ( \pm )-4 was followed with compound D $(0.230 \mathrm{~g}, 0.701 \mathrm{mmol})$ and TBAF $(2.80 \mathrm{~mL}, 1 \mathrm{M}$ in THF), giving the alkynyl alcohol ( \pm )-6 as a colorless oil $\left(0.158 \mathrm{~g}, 92 \%\right.$ yield). ${ }^{1} \mathrm{H}$ NMR $\left(400 \mathrm{MHz}, \mathrm{CDCl}_{3}\right) \delta 7.38-7.30(\mathrm{~m}$, $5 \mathrm{H}), 5.59(\mathrm{~d}, J=8.0 \mathrm{~Hz}, 1 \mathrm{H}), 5.11(\mathrm{~s}, 2 \mathrm{H}), 4.71(\mathrm{~m}, 1 \mathrm{H}), 4.01(\mathrm{~m}, 1 \mathrm{H}), 2.97(\mathrm{~d}, J=4.0$ $\mathrm{Hz}, 1 \mathrm{H}), 1 \mathrm{H}), 2.32(\mathrm{~d}, J=2.4 \mathrm{~Hz}, 1 \mathrm{H}), 1.83-1.70(\mathrm{~m}, 2 \mathrm{H}) 1.21(\mathrm{~d}, J=6.4 \mathrm{~Hz}, 3 \mathrm{H}) ;{ }^{13} \mathrm{C}$ NMR $\left(100 \mathrm{MHz}, \mathrm{CDCl}_{3}\right) \delta 156.59,136.27,128.76,128.49,128.42,82.88,71.89,64.46$, 66.44, 44.96, 41.24, 23.41; IR (neat) 3390 (brs), 1704, 1537, $1256 \mathrm{~cm}^{-1}$; HRMS [M+H] Calcd. for $\mathrm{C}_{14} \mathrm{H}_{18} \mathrm{O}_{3} \mathrm{~N}_{1}, 248.12812$, Found. 248.12809.

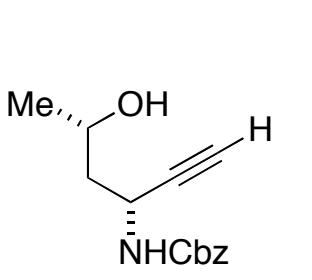

$( \pm)-6$

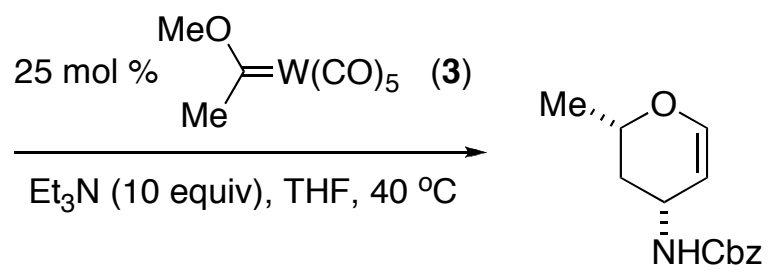

$( \pm)-7$

Synthesis of glycal ( $\mathbf{\pm}$-7: Following the general procedure for alkynyl alcohol cycloisomerization, substrate $( \pm)-6(25 \mathrm{mg}, 0.1 \mathrm{mmol})$ afforded glycal $( \pm)-7$ as a white crystal $\left(24 \mathrm{mg}, 95 \%\right.$ yield). MP $=92-93{ }^{\circ} \mathrm{C} ;{ }^{1} \mathrm{H}$ NMR $\left(400 \mathrm{MHz}, \mathrm{CDCl}_{3}\right) \delta 7.36-7.30(\mathrm{~m}$, $5 \mathrm{H}), 6.38(\mathrm{dd}, J=1.2,6.0 \mathrm{~Hz}, 1 \mathrm{H}), 5.10(\mathrm{~s}, 2 \mathrm{H}), 4.65(\mathrm{~d}, J=7.2 \mathrm{~Hz}, 1 \mathrm{H}), 4.58(\mathrm{dt}, J=$ 
2.0, $6.4 \mathrm{~Hz}, 1 \mathrm{H}$ ), 4.30 (ddd, $J=8.8 \mathrm{~Hz}, 1 \mathrm{H}), 4.08(\mathrm{dqd}, J=6.0 \mathrm{~Hz}, 1 \mathrm{H}), 2.26$ (dd, $J=6.4$, $12.8 \mathrm{~Hz}, 1 \mathrm{H}$ ), 1.41 (ddd, $J=11.2,13.2 \mathrm{~Hz}, 1 \mathrm{H}) 1.28$ (d, $J=6.4 \mathrm{~Hz}, 3 \mathrm{H}$ ); ${ }^{13} \mathrm{C}$ NMR $(100$ $\left.\mathrm{MHz}, \mathrm{CDCl}_{3}\right) \delta 156.09,146.27,136.65,128.77,128.39,102.58,71.77,66.92,44.26$, 37.59, 21.18; IR (KBr) 1683, 1234, $746 \mathrm{~cm}^{-1}$; HRMS [M+H] Calcd. for $\mathrm{C}_{14} \mathrm{H}_{18} \mathrm{O}_{3} \mathrm{~N}_{1}$, 248.12812, Found. 248.12801.

\section{Preparation of alkynyl alcohol 8}

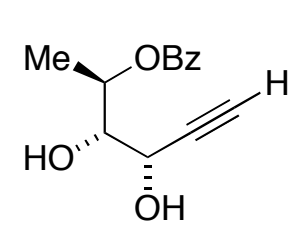

E

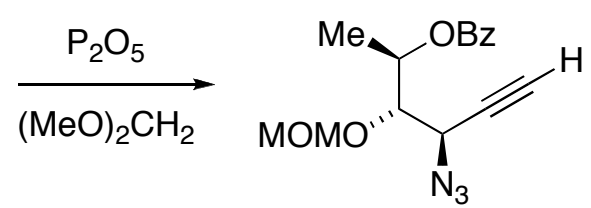

H

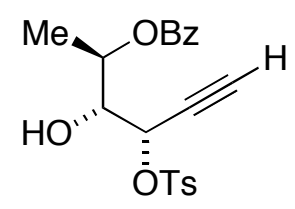

$\mathbf{F}$

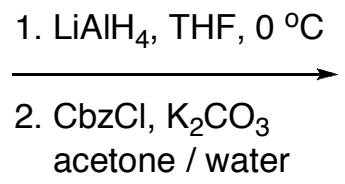

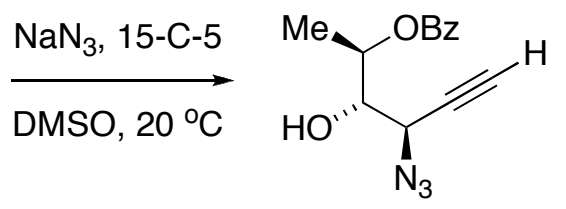

G

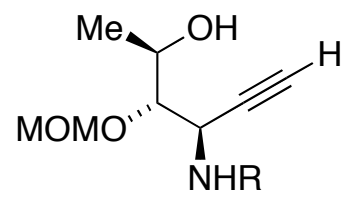

$\mathrm{I} \mathrm{R}=\mathrm{H}$

$8 \mathrm{R}=\mathrm{Cbz}$

Selective tosylation: A Dean-Stark column was fitted into a $50 \mathrm{~mL}$ round bottom flask containing a solution of known diol $\mathrm{E}^{2}(1.00 \mathrm{~g}, 4.27 \mathrm{mmol})$ and dibutyltin oxide $(1.34 \mathrm{~g}$, $1.34 \mathrm{mmol})$ in toluene $(20 \mathrm{~mL})$. The reaction mixture was refluxed for $5 \mathrm{~h}$, and then cooled to room temperature. $p$-Toluenesulfonyl chloride $(1.22 \mathrm{~g}, 6.40 \mathrm{mmol})$ was added, and the reaction mixture was stirred vigorously for $24 \mathrm{~h}$ at room temperature. Solvent was removed by rotary evaporation, and chromatography (hexanes : EtOAc $=4: 1$ ) gave monotosylated compound $\mathbf{F}$ as a colorless oil $(1.08 \mathrm{~g}, 65 \%$ yield $) .[\alpha]^{23}{ }_{D}=+39.2(\mathrm{c}$ = 2.07, $\left.\mathrm{CHCl}_{3}\right) ;{ }^{1} \mathrm{H} \mathrm{NMR}\left(400 \mathrm{MHz}, \mathrm{CDCl}_{3}\right) \delta$ 8.02-7.99 (m, 2H), 7.82-7.80 (m, 2H), 7.59 $(\mathrm{dt}, J=2.4,7.6 \mathrm{~Hz}, 1 \mathrm{H}), 7.46(\mathrm{dt}, J=1.6,8.0 \mathrm{~Hz}, 2 \mathrm{H}), 7.31(\mathrm{~d}, J=8.0 \mathrm{~Hz}, 2 \mathrm{H}), 5.31(\mathrm{dd}$, $J=2.0,3.6 \mathrm{~Hz}, 1 \mathrm{H}), 5.18(\mathrm{dt}, J=6.4,13.6 \mathrm{~Hz}, 1 \mathrm{H}), 4.11(\mathrm{dq}, J=3.6,6.4 \mathrm{~Hz}, 1 \mathrm{H}), 2.71$ $(\mathrm{d}, J=6.4 \mathrm{~Hz}, 1 \mathrm{H}), 2.46(\mathrm{~d}, J=2.4 \mathrm{~Hz}, 1 \mathrm{H}), 2.43(\mathrm{~s}, 3 \mathrm{H}), 1.46(\mathrm{~d}, J=6.4 \mathrm{~Hz}, 3 \mathrm{H}) ;{ }^{13} \mathrm{C}$ NMR $\left(100 \mathrm{MHz}, \mathrm{CDCl}_{3}\right) \delta 165.64,145.57,133.54,133.34,130.03,129.90,128.70$, 128.39, 78.95, 75.23, 75.18, 72.02, 71.16, 21.93, 16.65; IR (neat) 3503 (brs), 3281, 2127, 1717, 1275, $1177 \mathrm{~cm}^{-1}$; HRMS [M+H] Calcd. for $\mathrm{C}_{20} \mathrm{H}_{21} \mathrm{O}_{6}{ }^{32} \mathrm{~S}_{1} 389.10534$, Found 389.10454 .

(2) McDonald, F. E.; Reddy, K. S.; Díaz, Y. J. Am. Chem. Soc. 2000, 122, 4304. 
Azide substitution: The tosylate $\mathbf{F}(0.480 \mathrm{~g}, 1.24 \mathrm{mmol})$ was dissolved in dry DMSO (4 $\mathrm{mL})$, 15-Crown-5 $(0.4 \mathrm{~mL})$ and $\mathrm{NaN}_{3}(0.403 \mathrm{~g}, 6.20 \mathrm{mmol})$ were added, and the reaction mixture was stirred overnight at room temperature. The reaction mixture was diluted with $\mathrm{Et}_{2} \mathrm{O}(5 \mathrm{~mL})$ and quenched with water $(5 \mathrm{~mL})$. The organic layer was separated and the aqueous layer was extracted with $\mathrm{Et}_{2} \mathrm{O}(3 \times 5 \mathrm{~mL})$. The combined organic layer was dried over $\mathrm{MgSO}_{4}$ and solvent removed by rotary evaporation. Chromatography (hexanes : EtOAc $=5: 1)$ provided azido alcohol $\mathbf{G}$ as a white solid $(0.240 \mathrm{~g}, 75 \%$ yield). $\mathrm{MP}=69-70^{\circ} \mathrm{C} ;[\alpha]^{23}{ }_{\mathrm{D}}=-123.8\left(c=1.47, \mathrm{CHCl}_{3}\right) ;{ }^{1} \mathrm{H} \mathrm{NMR}\left(400 \mathrm{MHz}, \mathrm{CDCl}_{3}\right) \delta 8.05-8.01$ (m, 2H), $7.58(\mathrm{dt}, J=2.4,7.2 \mathrm{~Hz}, 1 \mathrm{H}), 7.47-7.43(\mathrm{~m}, 2 \mathrm{H}), 5.31(\mathrm{dq}, J=6.4,12.8 \mathrm{~Hz}, 1 \mathrm{H})$, 4.25 (dd, $J=2.4,5.2 \mathrm{~Hz}, 1 \mathrm{H}$ ), $3.97(\mathrm{t}, J=5.2 \mathrm{~Hz}, 1 \mathrm{H}$ ), $2.70(\mathrm{~d}, J=2.4 \mathrm{~Hz}, 1 \mathrm{H}), 2.58$ (brs, $1 \mathrm{H}), 1.46(\mathrm{~d}, J=6.4 \mathrm{~Hz}, 3 \mathrm{H}) ;{ }^{13} \mathrm{C}$ NMR $\left(100 \mathrm{MHz}, \mathrm{CDCl}_{3}\right) \delta 165.78,133.48,130.13$, 129.88, 128.68, 77.56, 76.52, 75.68, 70.95, 54.57, 15.93; IR (KBr) 3467 (brs), 3295, 2112, 1713, 1275, $713 \mathrm{~cm}^{-1}$; HRMS [M+H] Calcd. for $\mathrm{C}_{13} \mathrm{H}_{14} \mathrm{O}_{3} \mathrm{~N}_{3} 260.10297$, Found 260.10251.

MOM protection: The azido alcohol $\mathbf{G}(0.216 \mathrm{~g}, 0.833 \mathrm{mmol})$ was dissolved in dimethoxymethane $(4 \mathrm{~mL})$, and $\mathrm{P}_{2} \mathrm{O}_{5}(0.125 \mathrm{~g}, 0.833 \mathrm{mmol})$ was added. The reaction mixture was stirred for $30 \mathrm{~min}$ at room temperature, and additional $\mathrm{P}_{2} \mathrm{O}_{5}(0.125 \mathrm{~g}, 0.833$ $\mathrm{mmol}$ ) was added and then stirred for $30 \mathrm{~min}$. The reaction mixture was diluted with $\mathrm{CH}_{2} \mathrm{Cl}_{2}(5 \mathrm{~mL})$ and poured into a solution of cold saturated aq. $\mathrm{NaHCO}_{3}(20 \mathrm{~mL})$. The organic layer was separated and the aqueous layer was extracted with $\mathrm{CH}_{2} \mathrm{Cl}_{2}(3 \times 10$ $\mathrm{mL}$ ). The combined organic layer was dried over $\mathrm{MgSO}_{4}$ and evaporated under reduced pressure. Chromatography (hexanes : EtOAc $=10: 1$ ) gave MOM-protected compound $\mathbf{H}$ as a colorless oil $(0.180 \mathrm{~g}, 71 \%$ yield $)$. $[\alpha]^{23}{ }_{\mathrm{D}}=-58.95\left(c=3.2, \mathrm{CHCl}_{3}\right) ;{ }^{1} \mathrm{H}$ NMR $(400$ $\left.\mathrm{MHz}, \mathrm{CDCl}_{3}\right) \delta$ 8.05-8.02 (m, 2H), $7.56(\mathrm{dt}, J=2.8,7.2 \mathrm{~Hz}, 1 \mathrm{H}), 7.47-7.42(\mathrm{~m}, 2 \mathrm{H}), 5.40$ (dq, $J=4.8,6.4 \mathrm{~Hz}, 1 \mathrm{H}), 4.93(\mathrm{~d}, J=6.8 \mathrm{~Hz}, 1 \mathrm{H}), 4.81(\mathrm{~d}, J=5.6 \mathrm{~Hz}, 1 \mathrm{H}), 4.20(\mathrm{dd}, J=$ 2.4, $5.2 \mathrm{~Hz}, 1 \mathrm{H}$ ), 4.00 (dd, $J=4.8,5.2 \mathrm{~Hz}, 1 \mathrm{H}), 3.44(\mathrm{~s}, 3 \mathrm{H}), 2.70(\mathrm{~d}, J=2.0 \mathrm{~Hz}, 1 \mathrm{H}$ ), 1.45 (d, $J=6.4 \mathrm{~Hz}, 3 \mathrm{H}) ;{ }^{13} \mathrm{C}$ NMR $\left(100 \mathrm{MHz}, \mathrm{CDCl}_{3}\right) \delta 165.70,133.35,130.24,129.81$, 128.62, 98.02, 80.56, 77.57, 76.79, 70.95, 56.62, 53.78, 15.54; IR (neat) 2112, 1720, 1273, $1037 \mathrm{~cm}^{-1}$; HRMS [M+H] Calcd. for $\mathrm{C}_{15} \mathrm{H}_{18} \mathrm{O}_{4} \mathrm{~N}_{3} 304.12918$, Found 304.12836.

Reduction of azide: The azide $\mathbf{H}(0.180 \mathrm{~g}, 0.590 \mathrm{mmol})$ was dissolved in dry THF (4 $\mathrm{mL})$ and the solution was cooled to $0{ }^{\circ} \mathrm{C}$. Lithium aluminum hydride $(1.80 \mathrm{~mL}, 1 \mathrm{M}$ in 
THF, $1.8 \mathrm{mmol}$ ) was added dropwise to the $0{ }^{\circ} \mathrm{C}$ solution, and the reaction mixture was stirred for $3 \mathrm{~h}$ at $0{ }^{\circ} \mathrm{C}$. The reaction mixture was quenched by careful addition of water $(0.1 \mathrm{~mL})$ and $15 \% \mathrm{NaOH}(0.3 \mathrm{~mL})$, and then stirred for $1 \mathrm{~h}$ at room temperature. The resulting clear solution with white solid was treated with $\mathrm{MgSO}_{4}$ and filtered through Celite. After rotary evaporation of the organic layer, the amino alcohol I was obtained as a yellowish oil, which was directly used for the next step without further purification.

Cbz protection: The procedure as described above for $( \pm)-4$ and ( \pm )-6 was followed with crude amino alcohol $\mathrm{I}, \mathrm{K}_{2} \mathrm{CO}_{3}(17.4 \mathrm{mg}, 0.126 \mathrm{mmol})$ and $\mathrm{CbzCl}(0.266 \mathrm{~mL}, 1.89$ $\mathrm{mmol})$ in a mixture of acetone $(3 \mathrm{~mL}) /$ water $(3 \mathrm{~mL})$, and gave alkynyl alcohol 8 as a colorless oil ( $0.120 \mathrm{~g}, 67 \%$ yield over 2 steps) after chromatography (hexanes : EtOAc $=5: 1) .[\alpha]^{23}{ }_{\mathrm{D}}=+21.4\left(c=1.4, \mathrm{CHCl}_{3}\right) ;{ }^{1} \mathrm{H} \mathrm{NMR}\left(400 \mathrm{MHz}, \mathrm{CDCl}_{3}\right) \delta 7.37-7.31(\mathrm{~m}, 5 \mathrm{H})$, $5.54(\mathrm{~d}, J=8.4 \mathrm{~Hz}, 1 \mathrm{H}), 4.88(\mathrm{~d}, J=6.8 \mathrm{~Hz}, 2 \mathrm{H}), 3.88(\mathrm{brd}, J=6.0 \mathrm{~Hz}, 1 \mathrm{H}), 3.50$ (dd, $J$ $=3.2,5.2 \mathrm{~Hz}, 1 \mathrm{H}), 3.42(\mathrm{~s}, 3 \mathrm{H}), 3.11(\mathrm{~d}, J=4.4 \mathrm{~Hz}, 1 \mathrm{H}), 2.33(\mathrm{~d}, J=3.6 \mathrm{~Hz}, 1 \mathrm{H}), 1.27$ $(\mathrm{d}, J=6.4 \mathrm{~Hz}, 3 \mathrm{H}) ;{ }^{13} \mathrm{C}$ NMR $\left(100 \mathrm{MHz}, \mathrm{CDCl}_{3}\right) \delta 156.55,136.19,128.78,128.53$, $128.45,98.21,84.38,81.43,72.63,67.62,67.08,56.68,44.61,19.55$; IR (neat) 3410 (brs), 2926, 1709, 1514, 1252, $1030 \mathrm{~cm}^{-1}$; HRMS [M+H] Calcd. for $\mathrm{C}_{16} \mathrm{H}_{22} \mathrm{O}_{5} \mathrm{~N}_{1}$ 308.14925, Found 308.14896.

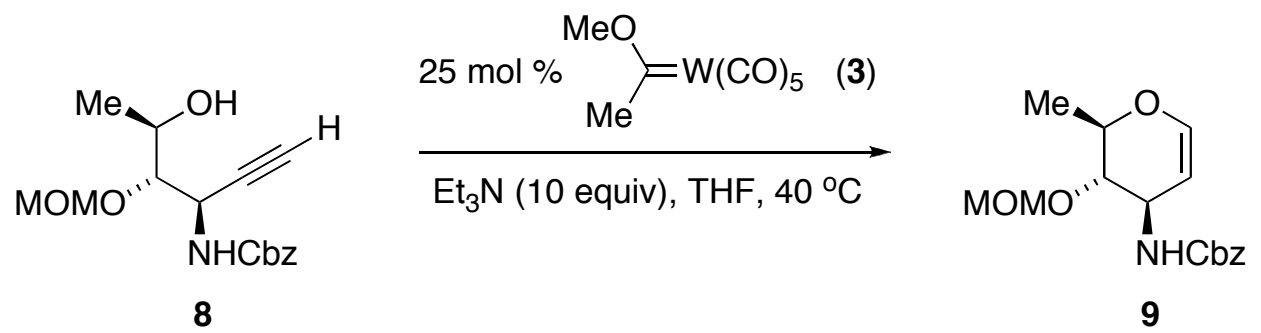

Synthesis of glycal 9: Following the general procedure for alkynyl alcohol cycloisomerization, substrate $8(30.7 \mathrm{mg}, 0.10 \mathrm{mmol})$ afforded glycal 9 as a white crystal $(29.4 \mathrm{mg}, 95 \%$ yield $) . \mathrm{MP}=102-103{ }^{\circ} \mathrm{C} ;[\alpha]^{23}{ }_{\mathrm{D}}=-74.2\left(c=0.99, \mathrm{CHCl}_{3}\right) ;{ }^{1} \mathrm{H} \mathrm{NMR}$ $\left(400 \mathrm{MHz}, \mathrm{CDCl}_{3}\right) \delta$ 7.36-7.30 (m, 5H), $6.31(\mathrm{dd}, J=1.6,6.4 \mathrm{~Hz}, 1 \mathrm{H}), 5.11(\mathrm{~s}, 2 \mathrm{H}), 5.06$ (brs, 1H), $4.73(\mathrm{~s}, 1 \mathrm{H}), 4.72(\mathrm{~d}, J=6.8 \mathrm{~Hz}, 1 \mathrm{H}), 4.67(\mathrm{~d}, J=6.8 \mathrm{~Hz}, 1 \mathrm{H}), 4.29(\mathrm{~m}, 1 \mathrm{H})$, 3.98 (dq, $J=6.4,8.4 \mathrm{~Hz}, 1 \mathrm{H}$ ), $3.36(\mathrm{dd}, J=8.4,1 \mathrm{H}$ ), $3.21(\mathrm{~s}, 3 \mathrm{H}), 1.35$ (d, $J=6.4 \mathrm{~Hz}$, $3 \mathrm{H}) ;{ }^{13} \mathrm{C}$ NMR $\left(100 \mathrm{MHz}, \mathrm{CDCl}_{3}\right) \delta 156.33,144.44,136.67,128.73,128.37,101.51$, 97.38, 78.85, 74.55, 66.94, 56.12, 50.36, 17.41; IR (KBr) 1693, 1553, 1234, $1047 \mathrm{~cm}^{-1}$; HRMS [M+H] Calcd. for $\mathrm{C}_{16} \mathrm{H}_{22} \mathrm{O}_{5} \mathrm{~N}_{1}, 308.14925$, Found. 308.14847. 


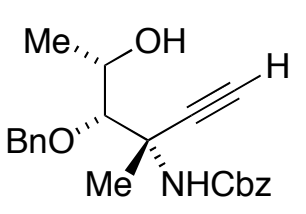

$( \pm)-10$

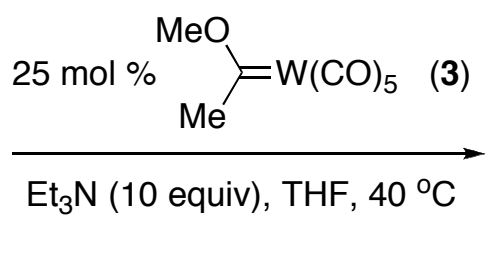

(3)

Synthesis of glycal $( \pm)-11$ : Following the general procedure for alkynyl alcohol cycloisomerization, the known alkynyl alcohol substrate $( \pm)-10$ (37 mg, $0.10 \mathrm{mmol})$ afforded known glycal ( \pm )-11 as a white crystal (31 mg, 84\% yield). All spectroscopic data for compounds $( \pm)-10$ and $( \pm)-11$ match the reported data. ${ }^{3}$

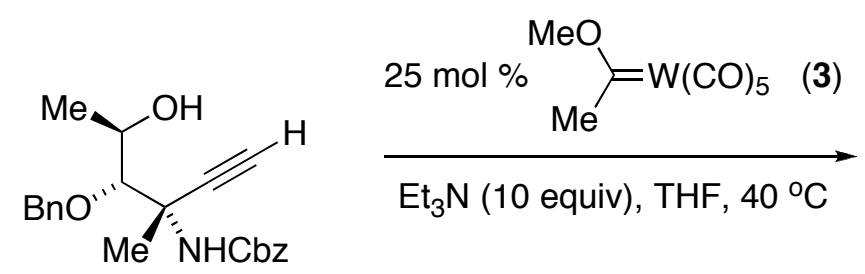

$( \pm)-12$

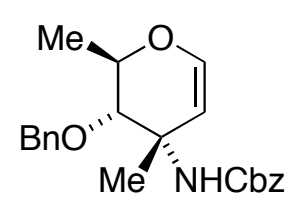

$( \pm)-13$

Synthesis of glycal (士)-13: Following the general procedure for alkynyl alcohol cycloisomerization, the known alkynyl alcohol substrate $( \pm)-12(37 \mathrm{mg}, 0.10 \mathrm{mmol})$ afforded known glycal ( \pm )-13 as a white crystal (35 mg, 94\% yield). All spectroscopic data for compounds $( \pm)-12$ and $( \pm)-13$ match the reported data. ${ }^{3}$

\section{Preparation of alkynyl alcohol 14}<smiles>C#C[C@@H](N)[C@@H](O)C(C)OC(C)=O</smiles>

G
1. $\mathrm{SnCl}_{2}, \mathrm{MeOH}, \mathrm{KF}$

2. $\mathrm{CbzCl}, \mathrm{K}_{2} \mathrm{CO}_{3}$ acetone / water

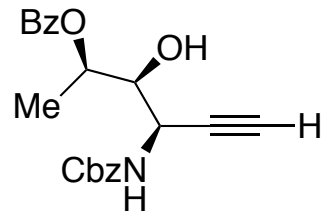

14

Reduction of azide: The azido alcohol $\mathbf{G}(0.16 \mathrm{~g}, 0.62 \mathrm{mmol})$ was dissolved in $\mathrm{MeOH}$ $(6 \mathrm{~mL})$, and $\mathrm{SnCl}_{2}(0.18 \mathrm{~g}, 0.93 \mathrm{mmol})$ was added. The reaction mixture was stirred at room temperature for $24 \mathrm{~h}$. Solvent was removed by rotary evaporation, and the crude material was dissolved in EtOAc $(6 \mathrm{~mL})$. Aqueous $\mathrm{KF}(3 \mathrm{~mL}, 5 \mathrm{M})$ was added and the reaction mixture was stirred at room temperature for $5 \mathrm{~h}$. The organic layer was

(3) Cutchins, W. W.; McDonald, F. E. Org. Lett. 2002, 4, 749. 
separated and the aqueous layer was extracted with EtOAc $(3 \times 5 \mathrm{~mL})$. The combined organic layer was dried over $\mathrm{MgSO}_{4}$, and solvents removed by rotary evaporation. The yellowish crude amino alcohol was used for the next step without further purification.

Cbz protection: The procedure as described above for $( \pm)-\mathbf{4},( \pm)-\mathbf{6}$ and $\mathbf{8}$ was followed with crude amino alcohol, $\mathrm{K}_{2} \mathrm{CO}_{3}(8.7 \mathrm{mg}, 0.062 \mathrm{mmol})$ and $\mathrm{CbzCl}(0.13 \mathrm{~mL}, 0.93 \mathrm{mmol})$ in a mixture of acetone $(2 \mathrm{~mL}) /$ water $(2 \mathrm{~mL})$, and gave the alkynyl alcohol 14 as a colorless oil $(0.146 \mathrm{~g}, 64 \%$ yield over 2 steps) after chromatography (hexanes : EtOAc $=$ $5: 1) .[\alpha]^{23}=+45.4\left(c=2.1, \mathrm{CHCl}_{3}\right) ;{ }^{1} \mathrm{H}$ NMR $\left(400 \mathrm{MHz}, \mathrm{CDCl}_{3}\right) \delta 8.02(\mathrm{~d}, J=7.6 \mathrm{~Hz}$, 2H), 7.55 (dt, $J=2.8,7.6 \mathrm{~Hz} 1 \mathrm{H}$ ), 7.43-7.39 (m, 2H), 7.31 (brs, $5 \mathrm{H}), 5.41$ (d, $J=9.2 \mathrm{~Hz}$, 1H), 5.24 (ddd, $J=2.4,4.8 \mathrm{~Hz}, 1 \mathrm{H}), 5.05(\mathrm{~s}, 2 \mathrm{H}), 4.75(\mathrm{~d}, J=3.2 \mathrm{~Hz}, 1 \mathrm{H}), 3.98(\mathrm{~d}, J=$ $4.4 \mathrm{~Hz}, 1 \mathrm{H}), 3.07(\mathrm{~d}, J=3.6 \mathrm{~Hz}, 1 \mathrm{H}), 2.39(\mathrm{~d}, J=2.0 \mathrm{~Hz}, 1 \mathrm{H}), 1.45(\mathrm{~d}, J=6.4 \mathrm{~Hz}, 3 \mathrm{H}$ ); ${ }^{13} \mathrm{C} \mathrm{NMR}\left(100 \mathrm{MHz}, \mathrm{CDCl}_{3}\right) \delta 166.02,156.12,136.14,133.35,130.21,129.86,128.71$, 128.62, 128.41, 80.95, 75.74, 73.18, 71.03, 67.52, 45.57, 16.28; IR (neat) 3406 (brs), 2926, 2252, 2120, 1968, 1713, 1524, 1275, $1116 \mathrm{~cm}^{-1}$; HRMS [M+H] Calcd. for $\mathrm{C}_{21} \mathrm{H}_{22} \mathrm{O}_{5} \mathrm{~N}_{1}$ 368.14925, Found 368.14822.
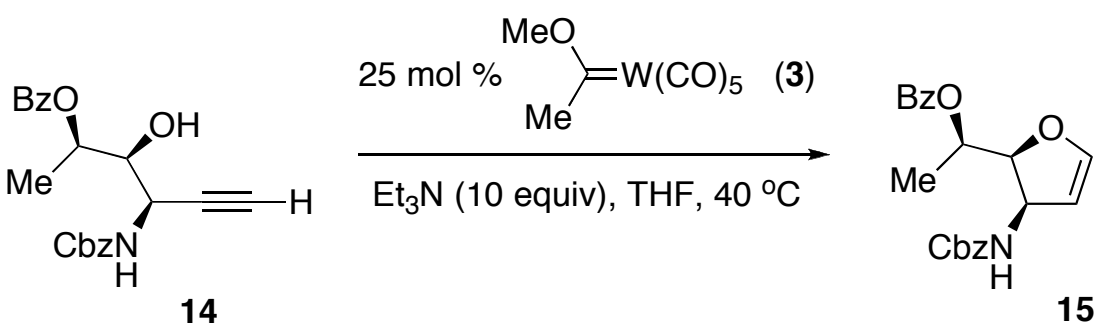

Synthesis of glycal 15: Following the general procedure for alkynyl alcohol cycloisomerization, substrate $14(37 \mathrm{mg}, 0.10 \mathrm{mmol})$ afforded glycal 15 as colorless oil (28 mg, 77\% yield). [a $]^{23}{ }_{\mathrm{D}}=+77.3\left(c=1.49, \mathrm{CHCl}_{3}\right) ;{ }^{1} \mathrm{H}$ NMR $\left(400 \mathrm{MHz}, \mathrm{CDCl}_{3}\right) \delta 8.04-$ $8.02(\mathrm{~m}, 2 \mathrm{H}), 7.55(\mathrm{dt}, J=2.8,7.6 \mathrm{~Hz}, 1 \mathrm{H}), 7.42(\mathrm{t}, J=7.6 \mathrm{~Hz}, 2 \mathrm{H}), 7.33-7.28(\mathrm{~m}, 3 \mathrm{H})$, 7.23-7.22 (m, 2H), $6.52(\mathrm{dd}, J=1.2,2.4 \mathrm{~Hz}, 1 \mathrm{H}), 5.46(\mathrm{dq}, J=6.4 \mathrm{~Hz}, 1 \mathrm{H}), 5.18-5.14(\mathrm{~m}$, $1 \mathrm{H}), 5.08(\mathrm{t}, J=3.2 \mathrm{~Hz}, 1 \mathrm{H}), 4.85(\mathrm{~d}, J=12.4 \mathrm{~Hz}, 1 \mathrm{H}), 4.76(\mathrm{~d}, J=10.0 \mathrm{~Hz}, 1 \mathrm{H}), 4.66(\mathrm{~d}$, $J=12.4 \mathrm{~Hz}, 1 \mathrm{H}), 4.44(\mathrm{dd}, J=7.6 \mathrm{~Hz}, 1 \mathrm{H}), 1.48(\mathrm{~d}, J=6.4 \mathrm{~Hz}, 3 \mathrm{H}) ;{ }^{13} \mathrm{C}$ NMR $(100 \mathrm{MHz}$, $\left.\mathrm{CDCl}_{3}\right) \delta$ 166. 12, 155.50, 149.45, 136.74, 133.55, 130.69, 130.32, 129.01, 128.69, 128.65, 102.98, 84.59, 68.71, 67.35, 54.50, 18.09; IR (KBr) 2925, 1716, 1524, 1266, 1059, $712 \mathrm{~cm}^{-1}$; HRMS [M+H] Calcd. for $\mathrm{C}_{21} \mathrm{H}_{22} \mathrm{O}_{5} \mathrm{~N}_{1}$ 368.14925, Found 368.14801. 


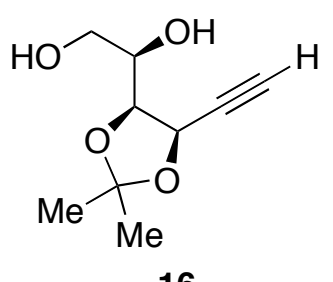

16

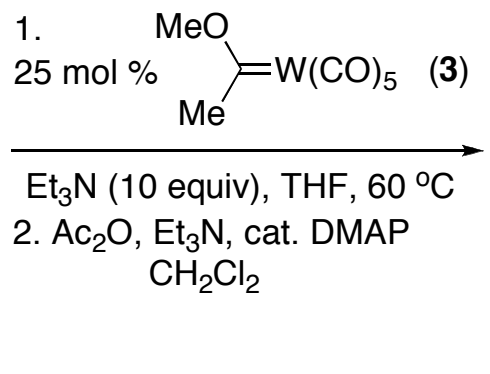

(3)

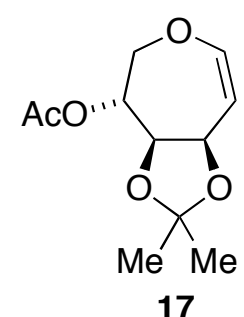

17

Synthesis of glycal 17: Following the general procedure for alkynyl alcohol cycloisomerization, the known substrate $16(15 \mathrm{mg}, 0.10 \mathrm{mmol})$ underwent reaction at $60{ }^{\circ} \mathrm{C}$. Upon completion, volatile components were removed by rotary evaporation. The crude mixture was dissolved in dry $\mathrm{CH}_{2} \mathrm{Cl}_{2}(1.5 \mathrm{~mL})$, and acetic anhydride (19 $\mu \mathrm{L}$, $0.2 \mathrm{mmol}$ ), triethylamine (62 $\mu \mathrm{L}, 0.4 \mathrm{mmol})$, and dimethylaminopyridine ( 1 crystal) were added, and the reaction mixture was stirred for $3 \mathrm{~h}$ at room temperature. After removal of volatiles by rotary evaporation, chromatography of the crude material (hexanes : EtOAc $=20: 1)$ gave the known glycal 17 as a white crystal $(15 \mathrm{mg}, 82 \%$ yield over two steps). All spectroscopic data for compounds 16 and 17 match with reported data. ${ }^{4}$

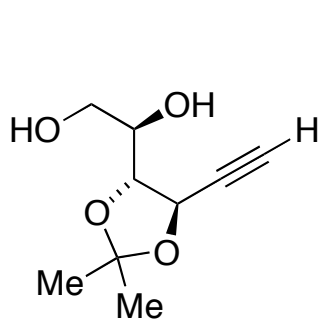

18

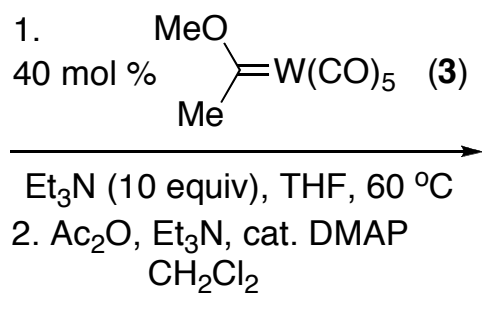

Synthesis of glycal 19: Following the general procedure for alkynyl alcohol cycloisomerization, the known substrate $18(15 \mathrm{mg}, 0.10 \mathrm{mmol})$ underwent reaction using $40 \mathrm{~mol} \%$ of 3 at $60{ }^{\circ} \mathrm{C}$. Acetylation and chromatographic purification (as described above for glycal 17) gave the known glycal 19 as a white crystal (15 mg, 82\% yield over two steps). All spectroscopic data for compounds 18 and 19 match with reported data. ${ }^{4}$

(4) Alcázar, E.; Pletcher, J. M.; McDonald, F. E. Org. Lett. 2004, 6, 3877. 


\section{Comparisons of non-photochemical procedure vs. photochemical procedures}

Condition A: The alkynyl alcohol substrate $(0.10 \mathrm{mmol})$ was dissolved in dry THF $(1.0$ $\mathrm{mL})$ in a conical vial. $\mathrm{Et}_{3} \mathrm{~N}(0.14 \mathrm{~mL}, 1.0 \mathrm{mmol})$ and tungsten Fischer carbene 3 (15.3 $\mathrm{mg}, 0.040 \mathrm{mmol}$ ) were added under argon atmosphere. The vial was sealed with a Teflon cap and then stirred for $24 \mathrm{~h}$ at $60^{\circ} \mathrm{C}$, after which time the reaction mixture was cooled to room temperature and solvent was removed by rotary evaporation. Chromatography (hexanes : EtOAc $=20: 1$ with $1 \% \mathrm{Et}_{3} \mathrm{~N}$ ) gave pure product.

Condition B: The alkynyl alcohol $(0.10 \mathrm{mmol})$ was dissolved in dry THF $(1 \mathrm{~mL})$ with stirring, and $\mathrm{W}(\mathrm{CO})_{6}(8.8 \mathrm{mg}, 0.025 \mathrm{mmol})$ and $\mathrm{Et}_{3} \mathrm{~N}(0.14 \mathrm{~mL}, 1.0 \mathrm{mmol})$ were added. The flask was fitted with a reflux condenser and then placed into Rayonet Photoreactor under an atmosphere of argon. The reaction mixture was irradiated at $350 \mathrm{~nm}$ at $60{ }^{\circ} \mathrm{C}$ for $6 \mathrm{~h}$, with stirring. Solvent was removed by rotary evaporation, and chromatography (hexanes : $\mathrm{EtOAc}=20: 1$ with $1 \% \mathrm{Et}_{3} \mathrm{~N}$ ) gave pure product.

Condition C: Condition B, except DABCO (22 mg, $0.20 \mathrm{mmol})$ was used instead of $\mathrm{Et}_{3} \mathrm{~N}$.

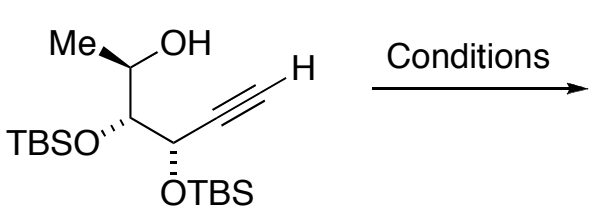

$20 a$

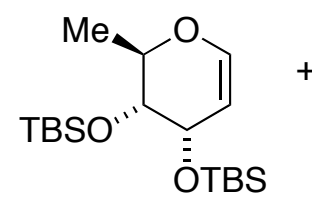

$21 \mathrm{a}$

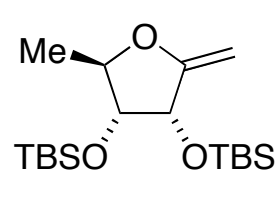

$22 a$

Cycloisomerizations of alkynyl alcohol 20a: Following the general procedure for alkynyl cycloisomerization with the conditions described above, the known alkynyl alcohol 20a (36 mg, $0.10 \mathrm{mmol}$ ) afforded known endo glycal 21a. ${ }^{2}$

Condition A: 21a (19 mg, 53\% yield) and 20a (11 mg, 30\%) was recovered Condition B: 21a (33 mg, 92\% yield).

All spectroscopic data for compounds $20 \mathrm{a}$ and $21 \mathrm{a}$ match the reported data. ${ }^{2}$ 


\section{Preparation of alkynyl alcohol 20b}

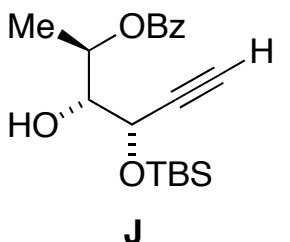

$\underset{(\mathrm{MeO})_{2} \mathrm{CH}_{2}}{\stackrel{\mathrm{P}_{2} \mathrm{O}_{5}}{\longrightarrow}}$

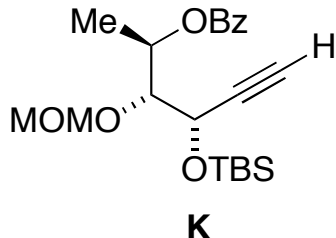

K

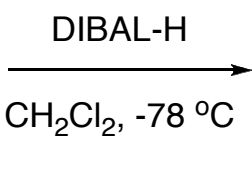

MOMO

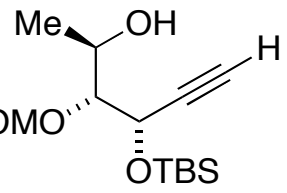

20b

MOM protection: The procedure as described above for alkynyl alcohol $\mathbf{8}$ was followed with known mono-TBS protected alkynyl alcohol $\mathbf{J}^{2}(0.28 \mathrm{~g}, 0.80 \mathrm{mmol})$ and $\mathrm{P}_{2} \mathrm{O}_{5}(0.23$ $\mathrm{g}, 1.60 \mathrm{mmol})$ in DMM (3 $\mathrm{mL})$, and produced MOM-protected product $\mathbf{K}$ as a colorless oil $(0.23 \mathrm{~g}, 73 \%$ yield) after chromatography (hexanes : EtOAc $=15: 1) .[\alpha]_{D}^{23}=+34.3$ $\left(c=0.7, \mathrm{CHCl}_{3}\right) ;{ }^{1} \mathrm{H}$ NMR $\left(400 \mathrm{MHz}, \mathrm{CDCl}_{3}\right) \delta 8.06-8.03(\mathrm{~m}, 2 \mathrm{H}), 7.58-7.54(\mathrm{~m}, 1 \mathrm{H})$, 7.46-7.42 (m, 2H), $5.43(\mathrm{dq}, J=4.0,6.4 \mathrm{~Hz}, 1 \mathrm{H}), 4.96(\mathrm{~d}, J=6.4 \mathrm{~Hz}, 1 \mathrm{H}), 4.81(\mathrm{~d}, J=$ $6.8 \mathrm{~Hz}, 1 \mathrm{H}), 4.51$ (dd, J = 2.4, $5.6 \mathrm{~Hz}, 1 \mathrm{H}), 3.96(\mathrm{dd}, J=4.0,5.6 \mathrm{~Hz}, 1 \mathrm{H}), 3.43(\mathrm{~s}, 3 \mathrm{H})$, 2.46 (d, J = 2.4 Hz, 1H), 1.43 (d, J = 6.4 Hz, 3H), 0.92 (s, 9H), 0.17 (s, 3H), 0.15 (s, 3H); ${ }^{13} \mathrm{C} \mathrm{NMR}\left(100 \mathrm{MHz}, \mathrm{CDCl}_{3}\right) \delta 165.81,133.15,130.68,129.83,128.57,97.72,82.83$, $81.43,74.41,71.20,64.30,56.46,25.91,18.33,15.24,-4.26,-5.03$; IR (neat) 2954, 2931, 2894, 2858, 2116, 1721, 1274, $839 \mathrm{~cm}^{-1}$; HRMS [M+Na] Calcd. for $\mathrm{C}_{21} \mathrm{H}_{32} \mathrm{O}_{5}{ }^{23} \mathrm{Na}_{1}{ }^{28} \mathrm{Si}_{1} 415.19112$, Found 415.19090.

Alkynyl alcohol 20b: The MOM-protected alkyne K (0.23 g, $0.59 \mathrm{mmol})$ was dissolved in dry $\mathrm{CH}_{2} \mathrm{Cl}_{2}(3 \mathrm{~mL})$, and cooled to $-78{ }^{\circ} \mathrm{C}$. DIBAL-H $(1.2 \mathrm{~mL}, 1.2 \mathrm{mmol})$ was added dropwise at $-78{ }^{\circ} \mathrm{C}$, and the reaction mixture was stirred for $1 \mathrm{hr}$ at $-78^{\circ} \mathrm{C}$. Cold EtOAc $(6 \mathrm{~mL})$ was added to quench the reaction, followed by Rochelle's salt $(5 \mathrm{~mL})$. The reaction mixture was stirred until two layers were clearly separated. The organic layer was separated and the aqueous layer was extracted with EtOAc $(3 \times 5 \mathrm{~mL})$. The combined organic layer was dried over $\mathrm{MgSO}_{4}$ and solvent removed by rotary evaporation. Chromatography (hexanes : EtOAc = $10: 1$ ) gave alkynyl alcohol 20b as a colorless oil $(0.12 \mathrm{~g}, 71 \%$ yield $) .[\alpha]^{23}=+105.8\left(c=2.3, \mathrm{CHCl}_{3}\right) ;{ }^{1} \mathrm{H} \mathrm{NMR}(400 \mathrm{MHz}$, $\left.\mathrm{CDCl}_{3}\right) \delta 4.82(\mathrm{~d}, J=6.4 \mathrm{~Hz}, 1 \mathrm{H}), 4.74(\mathrm{~d}, J=6.4 \mathrm{~Hz}, 1 \mathrm{H}), 4.40(\mathrm{dd}, J=2.4,6.0 \mathrm{~Hz}, 1 \mathrm{H})$, $3.98(\mathrm{dq}, J=6.4,9.6 \mathrm{~Hz}, 1 \mathrm{H}), 3.59(\mathrm{dd}, J=4.4,6.4 \mathrm{~Hz}, 1 \mathrm{H}), 3.43(\mathrm{~s}, 3 \mathrm{H}), 2.96(\mathrm{~d}, J=$ $8.0 \mathrm{~Hz}, 1 \mathrm{H}), 2.44$ (d, J = 2.4 Hz, 1H), 1.20 (d, J = 6.4 Hz, 3H), 0.88 (s, 9H), 0.15 (s, 3H), 0.11 (s, 3H); ${ }^{13} \mathrm{C}$ NMR (100 MHz, $\left.\mathrm{CDCl}_{3}\right) \delta 98.59,86.56,83.47,73.97,67.08,64.40$, 56.41, 25.84, 18.22, 18.14, -4.22, -5.09; IR (neat) 3454 (brs), 3310, 2955, 2931, 2897, 2858, 2115, 1253, 1153, 1104, 1033, $839 \mathrm{~cm}^{-1}$; HRMS Calcd. [M+H] for $\mathrm{C}_{14} \mathrm{H}_{29} \mathrm{O}_{4}{ }^{28} \mathrm{Si}_{1}$ 289.18296, Found 289.18288. 

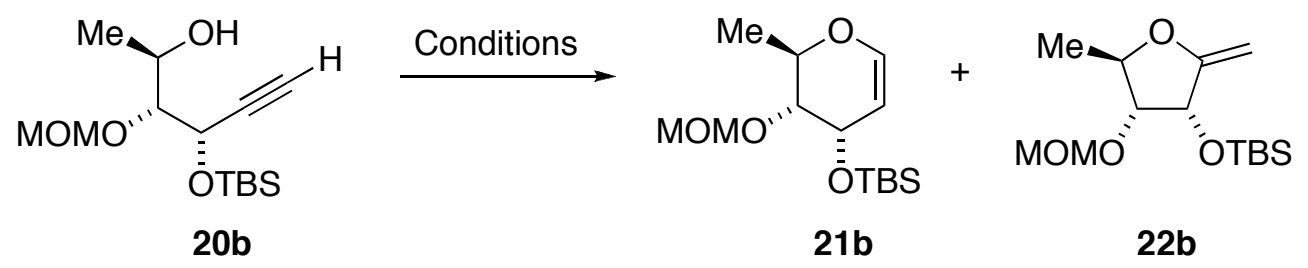

Cycloisomerizations of alkynyl alcohol 20b: Following the conditions described for cycloisomerization of 20a, the alkynyl alcohol substrate $20 \mathrm{~b}(29 \mathrm{mg}, 0.10 \mathrm{mmol})$ afforded endo glycal $\mathbf{2 1 b}$ and exo product 22b.

Condition A: 21b (15 mg, 53\% yield) and 22b (10 mg, 35\% yield).

Condition B: 21b (21 mg, 65\% yield) and 22b (6.6 mg, 20\% yield).

Condition C: 21b (22 mg, 75\% yield) and 22b (4.4 mg, 15\% yield).

21b: $[\alpha]^{23}{ }_{D}=+297.2\left(c=1.48, \mathrm{CHCl}_{3}\right) ;{ }^{1} \mathrm{H}$ NMR $\left(400 \mathrm{MHz}, \mathrm{CDCl}_{3}\right) \delta 6.31(\mathrm{~d}, J=6.0 \mathrm{~Hz}$, $1 \mathrm{H}), 4.81(\mathrm{~d}, J=6.4 \mathrm{~Hz}, 1 \mathrm{H}), 4.76(\mathrm{dd}, J=6.0 \mathrm{~Hz}, 1 \mathrm{H}), 4.61(\mathrm{~d}, J=6.4 \mathrm{~Hz}, 1 \mathrm{H}), 4.25$ (dd, $J=3.6,5.2 \mathrm{~Hz}, 1 \mathrm{H}$ ), $4.17(\mathrm{dq}, J=3.6,6.4 \mathrm{~Hz}, 1 \mathrm{H}$ ), 3.51 (dd, $J=3.2,9.2 \mathrm{~Hz}, 1 \mathrm{H}$ $3.40(\mathrm{~s}, 3 \mathrm{H}), 3.34(\mathrm{~d}, J=6.4 \mathrm{~Hz}), 1.28(\mathrm{~d}, J=6.4 \mathrm{~Hz}, 3 \mathrm{H}), 0.89(\mathrm{~s}, 9 \mathrm{H}), 0.08(\mathrm{~s}, 3 \mathrm{H})$, 0.07 (s, 3H); ${ }^{13} \mathrm{C}$ NMR $\left(100 \mathrm{MHz}, \mathrm{CDCl}_{3}\right) \delta 145.17,101.98,94.72,76.13,70.01,61.17$, 55.90, 26.08, 18.39, 17.84, -3.75, -4.26; IR (neat) 2953, 2929, 2857, 1642, 1472, 1240 , 1149, 1119, $835 \mathrm{~cm}^{-1}$; HRMS Calcd. [M+H] for $\mathrm{C}_{14} \mathrm{H}_{29} \mathrm{O}_{4}{ }^{28} \mathrm{Si}_{1}$ 289.18296, Found 289.18292.

22b: ${ }^{1} \mathrm{H}$ NMR $\left(400 \mathrm{MHz}, \mathrm{CDCl}_{3}\right) \delta 4.74(\mathrm{~d}, J=7.2 \mathrm{~Hz}, 1 \mathrm{H}), 4.68(\mathrm{~d}, J=6.4 \mathrm{~Hz}, 1 \mathrm{H})$, $4.56(\mathrm{~d}, J=4.4 \mathrm{~Hz}, 1 \mathrm{H}), 4.38(\mathrm{dq}, J=5.2,6.4 \mathrm{~Hz}, 1 \mathrm{H}), 4.33(\mathrm{dd}, J=1.6 \mathrm{~Hz}, 1 \mathrm{H}), 4.05$ (dd, $J=1.2,1.6 \mathrm{~Hz}, 1 \mathrm{H}), 3.71(\mathrm{dd}, J=4.4 \mathrm{~Hz}, 1 \mathrm{H}), 3.39(\mathrm{~s}, 3 \mathrm{H}), 1.32(\mathrm{~d}, J=6.8 \mathrm{~Hz}, 3 \mathrm{H}$ ), 0.92 (s, 9H), 0.14 (s, 3H), 0.12 (s, 3H); IR (neat) 2917, 2853, 1462, $1052 \mathrm{~cm}^{-1}$; HRMS Calcd. [M+H] for $\mathrm{C}_{14} \mathrm{H}_{29} \mathrm{O}_{4}{ }^{28} \mathrm{Si}_{1} 289.18296$, Found 289.18285 . 


\section{Preparation of alkynyl alcohol 20c}

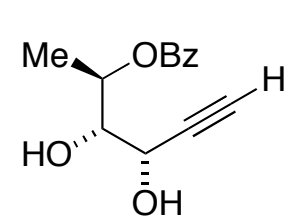

E

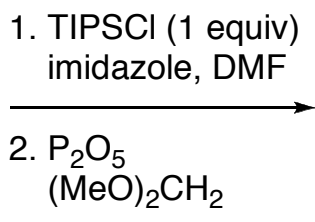

$(\mathrm{MeO})_{2} \mathrm{CH}_{2}$

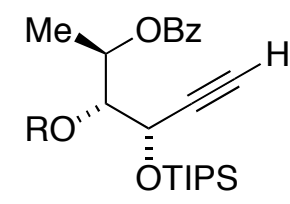

$L R=H$

M $\mathrm{R}=\mathrm{MOM}$

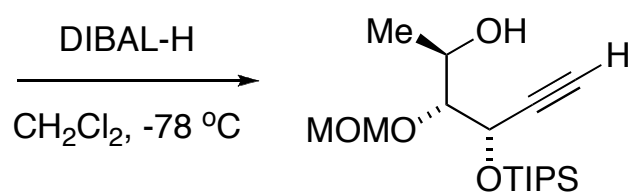

20c

Selective TIPS protection of diol: The known diol $E^{2}(1.06 \mathrm{~g}, 4.53 \mathrm{mmol})$ was dissolved in dry DMF $(5 \mathrm{~mL})$, imidazole $(0.616 \mathrm{~g}, 9.05 \mathrm{mmol})$ and TIPSCI $(1.05 \mathrm{~mL}, 4.98$ $\mathrm{mmol}$ ) were added, and the reaction mixture was stirred for $1 \mathrm{~h}$ at room temperature. The reaction was quenched with water $(3 \mathrm{~mL})$ and diluted with ethyl ether $(5 \mathrm{~mL})$. The organic layer was separated and the aqueous layer was extracted with ethyl ether $(3 \times 5$ $\mathrm{mL}$ ). The combined organic layer was dried over $\mathrm{MgSO}_{4}$ and evaporated under reduced pressure. Chromatography (hexanes : EtOAc $=15: 1$ ) gave mono-TIPS protected alkynyl alcohol $L$ as a colorless oil $(1.30 \mathrm{~g}, 73 \%$ yield $)$. $[\alpha]^{23}=-22.7\left(c=1.14, \mathrm{CHCl}_{3}\right)$; ${ }^{1} \mathrm{H}$ NMR $\left(400 \mathrm{MHz}, \mathrm{CDCl}_{3}\right)$ ठ 8.03-8.00 (m, 2H), $7.57(\mathrm{dt}, J=2.4,7.6 \mathrm{~Hz}, 1 \mathrm{H}), 7.44(\mathrm{t}, J$ $=7.6 \mathrm{~Hz}, 2 \mathrm{H}), 5.29(\mathrm{dq}, J=6.4,7.6 \mathrm{~Hz}, 1 \mathrm{H}), 4.72(\mathrm{dd}, J=2.4,3.6 \mathrm{~Hz}, 1 \mathrm{H}), 3.91$ (ddd, $J$ $=3.6,7.6 \mathrm{~Hz}, 1 \mathrm{H}), 2.67(\mathrm{~d}, J=3.6 \mathrm{~Hz}, 1 \mathrm{H}), 2.45(\mathrm{~d}, J=2.4 \mathrm{~Hz}, 1 \mathrm{H}), 1.49(\mathrm{~d}, J=6.4 \mathrm{~Hz}$, $3 \mathrm{H}), 1.22-1.03(\mathrm{~m}, 21 \mathrm{H}) ;{ }^{13} \mathrm{C}$ NMR $\left(100 \mathrm{MHz}, \mathrm{CDCl}_{3}\right) \delta 165.56,133.26,130.53,129.78$, 128.62, 80.98, 76.58, 75.29, 71.66, 64.78, 18.19, 18.17, 17.94, 16.71, 12.36; IR (neat) 3514 (brs), 3308, 2943, 2866, 1720, 1274, 1113, $1067 \mathrm{~cm}^{-1}$; HRMS [M+H] Calcd. for $\mathrm{C}_{22} \mathrm{H}_{35} \mathrm{O}_{4}{ }^{28} \mathrm{Si}_{1}$ 391.22991, Found 392.22853.

MOM protection: The procedure as described above for alkynyl alcohol 8 was followed with TIPS-protected alkynyl alcohol $\mathrm{L}(1.30 \mathrm{~g}, 3.33 \mathrm{mmol})$ and $\mathrm{P}_{2} \mathrm{O}_{5}(1.87 \mathrm{~g}, 6.66 \mathrm{mmol})$ in dimethoxymethane $(5 \mathrm{~mL})$, and gave MOM-protected alkyne $\mathbf{M}$ as a colorless oil $(1.25 \mathrm{~g}, 87 \%$ yield) after chromatography (hexanes : EtOAc $=15: 1) \cdot[\alpha]^{23}{ }_{\mathrm{D}}=+25.7(c=$ 2.6, $\left.\mathrm{CHCl}_{3}\right) ;{ }^{1} \mathrm{H}$ NMR $\left(400 \mathrm{MHz}, \mathrm{CDCl}_{3}\right) \delta 8.05-8.03(\mathrm{~m}, 2 \mathrm{H}), 7.56(\mathrm{dt}, J=2.8,7.6 \mathrm{~Hz}$, $1 \mathrm{H}), 7.43$ (t, $J=7.6 \mathrm{~Hz}, 2 \mathrm{H}), 5.41(\mathrm{dq}, J=6.4,7.2 \mathrm{~Hz}, 1 \mathrm{H}), 5.04(\mathrm{~d}, J=7.2 \mathrm{~Hz}, 1 \mathrm{H})$, $4.82(\mathrm{~d}, J=7.2 \mathrm{~Hz}, 1 \mathrm{H}), 4.69(\mathrm{dd}, J=2.0,4.4 \mathrm{~Hz}, 1 \mathrm{H}), 3.99(\mathrm{dd}, J=4.4,5.6 \mathrm{~Hz}, 1 \mathrm{H})$, 3.45 (s, 3H), $2.47(\mathrm{~d}, J=2.4 \mathrm{~Hz}, 1 \mathrm{H}), 1.46(\mathrm{~d}, J=6.4 \mathrm{~Hz}, 3 \mathrm{H}), 1.21-1.08(\mathrm{~m}, 21 \mathrm{H}) ;{ }^{13} \mathrm{C}$ NMR $\left(100 \mathrm{MHz}, \mathrm{CDCl}_{3}\right) \delta 165.70,133.17,130.60,129.83,128.54,97.77,82.49,81.76$, 74.66, 71.17, 64.87, 56.49, 18.23, 18.22, 15.97, 12.44; IR (neat) 2943, 2867, 1721, 1273, 1112, 1036, $712 \mathrm{~cm}^{-1}$; HRMS [M+H] Calcd. for $\mathrm{C}_{24} \mathrm{H}_{39} \mathrm{O}_{5}{ }^{28} \mathrm{Si}_{1} 435.25613$, Found 435.25507. 
Alkynyl alcohol 20c: The tri-O-protected alkyne $\mathbf{M}(1.25 \mathrm{~g}, 2.88 \mathrm{mmol})$ was dissolved in dry $\mathrm{CH}_{2} \mathrm{Cl}_{2}(10 \mathrm{~mL})$, cooled to $-78{ }^{\circ} \mathrm{C}$, and DIBAL-H $(5.76 \mathrm{~mL}, 5.76 \mathrm{mmol})$ was added dropwise at $-78{ }^{\circ} \mathrm{C}$. The reaction mixture was stirred for $1 \mathrm{~h}$ at $-78{ }^{\circ} \mathrm{C}$ after which time cold EtOAc $(30 \mathrm{~mL})$ was added to quench the reaction, followed by Rochelle's salt $(20$ $\mathrm{mL}$ ). The reaction mixture was stirred until two layers were clearly separated. The organic layer was separated and the aqueous layer was extracted with EtOAc (3 310 $\mathrm{mL}$ ). The combined organic layer was dried over $\mathrm{MgSO}_{4}$ and evaporated under reduced pressure. Chromatography (hexanes : EtOAc $=10: 1$ ) gave alkynyl alcohol 20c as a colorless oil $(0.699 \mathrm{~g}, 73 \%$ yield $)$. $[\alpha]^{23}{ }_{\mathrm{D}}=+89.7\left(c=0.73, \mathrm{CHCl}_{3}\right) ;{ }^{1} \mathrm{H} \mathrm{NMR}(400 \mathrm{MHz}$, $\left.\mathrm{CDCl}_{3}\right) \delta 4.89(\mathrm{~d}, J=6.4 \mathrm{~Hz}, 1 \mathrm{H}), 4.76(\mathrm{~d}, J=6.4 \mathrm{~Hz}, 1 \mathrm{H}), 4.62(\mathrm{dd}, J=2.4,5.6 \mathrm{~Hz}, 1 \mathrm{H})$, $4.02(\mathrm{dq}, J=6.4,12.6 \mathrm{~Hz}, 1 \mathrm{H}), 3.62(\mathrm{t}, J=4.8 \mathrm{~Hz}, 1 \mathrm{H}), 3.44(\mathrm{~s}, 3 \mathrm{H}), 2.73(\mathrm{~d}, J=7.2 \mathrm{~Hz}$, $1 \mathrm{H}), 2.47(\mathrm{~d}, J=2.4 \mathrm{~Hz}, 1 \mathrm{H}), 1.27(\mathrm{~d}, J=6.4 \mathrm{~Hz}, 3 \mathrm{H}), 1.21-1.08(\mathrm{~m}, 21 \mathrm{H}) ;{ }^{13} \mathrm{C} N M R$ $\left(100 \mathrm{MHz}, \mathrm{CDCl}_{3}\right) \delta 98.48,86.55,83.38,74.33,67.38,64.91,56.46,18.84,18.25$, 12.51; IR (neat) 3436 (brs), 3310, 2943, 2867, 2115, 1463, 1105, $1033 \mathrm{~cm}^{-1}$; HRMS Calcd. for $\mathrm{C}_{17} \mathrm{H}_{35} \mathrm{O}_{4}{ }^{28} \mathrm{Si}_{1} 331.22991$, Found 331.22895.

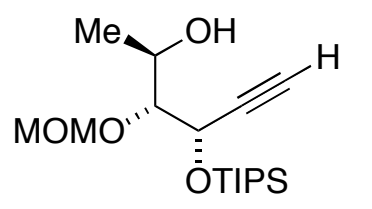

20c

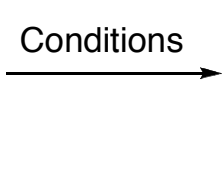

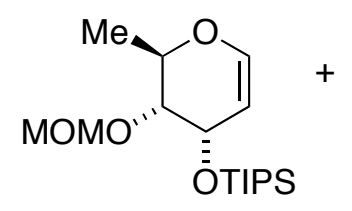

21c

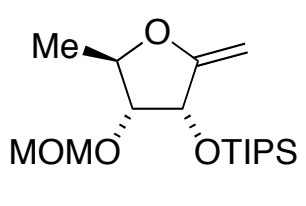

22c

Cycloisomerizations of alkynyl alcohol 20c: Following the conditions described for cycloisomerization of $20 \mathrm{a}$, the alkynyl alcohol substrate $20 \mathrm{c}(33 \mathrm{mg}, 0.10 \mathrm{mmol}$ ) afforded endo glycal 21c and exo product 22c.

Condition A: 21c (28 mg, 85\% yield) and 22c ( $3.0 \mathrm{mg}, 9 \%$ yield).

Condition B: 21c (29 mg, 88\% yield) and 22c (2.3 mg, 7\% yield).

Condition C: $21 \mathrm{c}(29 \mathrm{mg}, 88 \%$ yield $)$ and $22 \mathrm{c}(2.0 \mathrm{mg}, 6 \%$ yield $)$.

21c: $[\alpha]^{23}=+279.4\left(c=1.2, \mathrm{CHCl}_{3}\right) ;{ }^{1} \mathrm{H}$ NMR $\left(400 \mathrm{MHz}, \mathrm{CDCl}_{3}\right) \delta 6.31(\mathrm{~d}, J=6.0 \mathrm{~Hz}$, $1 \mathrm{H}), 4.85(\mathrm{~d}, J=6.8 \mathrm{~Hz}, 1 \mathrm{H}), 4.83(\mathrm{dd}, J=5.6,6.0 \mathrm{~Hz}, 1 \mathrm{H}), 4.63(\mathrm{~d}, J=7.2 \mathrm{~Hz}, 1 \mathrm{H})$, $4.39(\mathrm{dd}, J=3.6,5.2 \mathrm{~Hz}, 1 \mathrm{H}), 4.23(\mathrm{dq}, J=6.4,8.8 \mathrm{~Hz}, 1 \mathrm{H}), 3.54(\mathrm{dd}, J=3.6,8.8 \mathrm{~Hz}$, $1 \mathrm{H}), 3.41(\mathrm{~s}, 3 \mathrm{H}), 1.34(\mathrm{~d}, J=6.8 \mathrm{~Hz}, 3 \mathrm{H}), 1.07-1.06(\mathrm{~m}, 21 \mathrm{H}) ;{ }^{13} \mathrm{C}$ NMR $(100 \mathrm{MHz}$, 
$\left.\mathrm{CDCl}_{3}\right) \delta 145.07,101.97,94.85,76.27,70.19,61.32,55.92,18.37,17.90,12.93$; IR (neat) 2941, 1641, 1240, 1041, $1005 \mathrm{~cm}^{-1}$; HRMS [M+H] Calcd. for $\mathrm{C}_{17} \mathrm{H}_{35} \mathrm{O}_{4}{ }^{28} \mathrm{Si}_{1}$ 331.22991 , Found 331.22919 .

22c: $[\alpha]^{23}{ }_{\mathrm{D}}=-69.8\left(c=1.8, \mathrm{CHCl}_{3}\right) ;{ }^{1} \mathrm{H} \mathrm{NMR}\left(400 \mathrm{MHz}, \mathrm{CDCl}_{3}\right) \delta 4.77(\mathrm{~d}, J=6.8 \mathrm{~Hz}$, $1 \mathrm{H}), 4.70(\mathrm{~d}, J=2.0 \mathrm{~Hz}, 1 \mathrm{H}), 4.69(\mathrm{~d}, J=6.8 \mathrm{~Hz}, 1 \mathrm{H}), 4.40(\mathrm{dq}, J=4.8,6.8 \mathrm{~Hz}, 1 \mathrm{H})$, $4.33(\mathrm{dd}, J=1.6 \mathrm{~Hz}, 1 \mathrm{H}), 4.13(\mathrm{dd}, J=1.6 \mathrm{~Hz}, 1 \mathrm{H}), 3.71(\mathrm{dd}, J=4.4 \mathrm{~Hz}, 1 \mathrm{H}), 3.39$ (s, $3 \mathrm{H}), 1.31(\mathrm{~d}, J=6.4 \mathrm{~Hz}, 3 \mathrm{H}), 1.15-1.07(\mathrm{~m}, 21 \mathrm{H}) ;{ }^{13} \mathrm{C} \mathrm{NMR}\left(100 \mathrm{MHz}, \mathrm{CDCl}_{3}\right) \delta 162.18$, 96.26, 83.27, 81.22, 79.03, 71.45, 55.93, 18.98, 18.28, 18.26, 12.78; IR (neat) 2942, 2867, 1463, 1147, 1052, $996 \mathrm{~cm}^{-1}$; HRMS [M+H] Calcd. for $\mathrm{C}_{17} \mathrm{H}_{35} \mathrm{O}_{4}{ }^{28} \mathrm{Si}_{1} 331.22991$, Found 331.22979.

\section{Synthesis of the altromycin disaccharide 33}

\section{Enantiomer resolution of $( \pm)-28$}

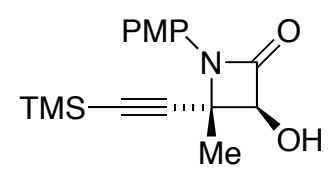

$( \pm)-28$

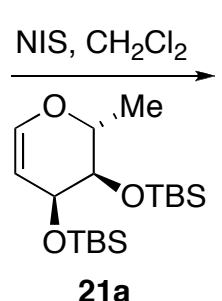

21a

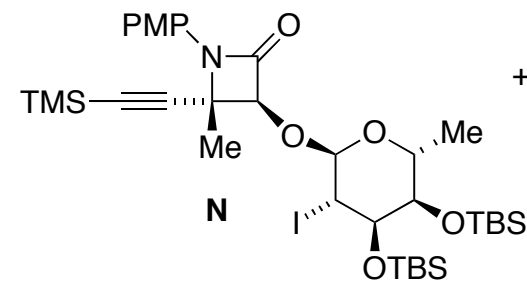
1. separate diastereomers
2. MeLi, THF, $-78^{\circ} \mathrm{C}$

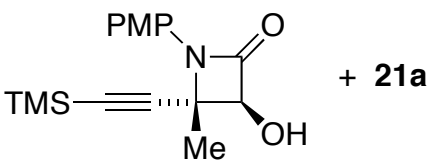

$(+)-28$

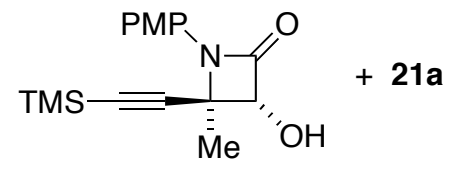

$(-)-28$

NIS mediated glycosylation: Racemic hydroxyl beta-lactam $( \pm)-28^{3}$ (2.25 g, 7.42 $\mathrm{mmol}$ ) and bis-TBS protected glycal 21a (2.40 g, $6.67 \mathrm{mmol})$ were dissolved in $\mathrm{CH}_{2} \mathrm{Cl}_{2}$ $(40 \mathrm{~mL})$, and cooled to $0{ }^{\circ} \mathrm{C}$. NIS $(1.59 \mathrm{~g}, 7.05 \mathrm{mmol})$ was added, and the mixture was slowly warmed to room temperature and stirred overnight at room temperature in the dark. The reaction mixture was diluted with $\mathrm{CH}_{2} \mathrm{Cl}_{2}(10 \mathrm{~mL})$ and quenched with sat. aq. $\mathrm{Na}_{2} \mathrm{~S}_{2} \mathrm{O}_{4}(20 \mathrm{~mL})$. The organic layer was separated and the aqueous layer was extracted with $\mathrm{CH}_{2} \mathrm{Cl}_{2}(3 \times 10 \mathrm{~mL})$. The combined organic layer was dried over $\mathrm{MgSO}_{4}$ 
and solvent was removed by rotary evaporation. Chromatography (hexanes: EtOAc $=$ $30: 1)$ gave each separable glycosylated beta-lactam $\mathbf{N}(2.50 \mathrm{~g}, 47 \%$ yield) as a foam and $\mathbf{O}(2.39 \mathrm{~g}, 45 \%$ yield $)$ as a colorless oil.

Compound N: $[\alpha]^{23}{ }_{D}=+114.22\left(c=6.6, \mathrm{CHCl}_{3}\right) ;{ }^{1} \mathrm{H} \mathrm{NMR}\left(400 \mathrm{MHz}, \mathrm{CDCl}_{3}\right) \delta 7.55(\mathrm{~d}, J$ $=8.8 \mathrm{~Hz}, 2 \mathrm{H}), 6.86(\mathrm{~d}, J=8.8 \mathrm{~Hz}, 2 \mathrm{H}), 5.19(\mathrm{~s}, 1 \mathrm{H}), 4.96(\mathrm{~s}, 1 \mathrm{H}), 4.63(\mathrm{brs}, 1 \mathrm{H}), 4.29(\mathrm{~d}$, $J=2.4 \mathrm{~Hz}, 1 \mathrm{H}), 4.15(\mathrm{~d}, J=10.0 \mathrm{~Hz}, 1 \mathrm{H}), 4.12(\mathrm{~s}, 1 \mathrm{H}), 3.78(\mathrm{~s}, 1 \mathrm{H}), 1.65(\mathrm{~s}, 3 \mathrm{H}), 1.30$ (d, $J=6.4 \mathrm{~Hz}, 3 \mathrm{H}), 0.91(\mathrm{~s}, 9 \mathrm{H}), 0.87(\mathrm{~s}, 9 \mathrm{H}), 0.16(\mathrm{~s}, 9 \mathrm{H}), 0.14(\mathrm{~s}, 3 \mathrm{H}), 0.10(\mathrm{~s}, 3 \mathrm{H})$, 0.07 (s, 6H); ${ }^{13} \mathrm{C}$ NMR $\left(100 \mathrm{MHz}, \mathrm{CDCl}_{3}\right) \delta 162.73,156.64,129.85,119.58,114.39$, 104.17, 102.43, 92.51, 86.57, 74.66, 70.08, 65.15, 57.47, 55.62, 29.27, 26.23, 25.92, 20.52, 18.23, 18.17, 17.98, -0.09, -3.42, -3.59, -4.34, -4.67; IR (neat) 2953, 2932, 2896, 2858, 2253, 2162, 1764, 1513, 1250, 1093, 976, $839 \mathrm{~cm}^{-1}$; HRMS [M+H] Calcd. for $\mathrm{C}_{34} \mathrm{H}_{59} \mathrm{O}_{6} \mathrm{~N}_{1}{ }^{127} \mathrm{I}_{1}{ }^{28} \mathrm{Si}_{3}$ 788.26895, Found 788.26923.

Compound O: $[\alpha]^{23}{ }_{\mathrm{D}}=+8.1\left(c=2.78, \mathrm{CHCl}_{3}\right) ;{ }^{1} \mathrm{H} \mathrm{NMR}\left(400 \mathrm{MHz}, \mathrm{CDCl}_{3}\right) \delta 7.57(\mathrm{~d}, J=$ $8.8 \mathrm{~Hz}, 2 \mathrm{H}), 6.88(\mathrm{~d}, J=9.2 \mathrm{~Hz}, 2 \mathrm{H}), 5.27(\mathrm{~s}, 1 \mathrm{H}), 4.84(\mathrm{~s}, 1 \mathrm{H}), 4.38(\mathrm{~d}, J=2.4 \mathrm{~Hz}, 1 \mathrm{H})$, $4.30(\mathrm{~m}, 1 \mathrm{H}), 4.16-4.13(\mathrm{~m}, 2 \mathrm{H}), 3.80(\mathrm{~s}, 1 \mathrm{H}), 1.67(\mathrm{~s}, 3 \mathrm{H}), 1.25(\mathrm{~d}, J=6.4 \mathrm{~Hz}, 3 \mathrm{H})$, $0.92(\mathrm{~s}, 9 \mathrm{H}), 0.88(\mathrm{~s}, 9 \mathrm{H}), 0.17(\mathrm{~s}, 9 \mathrm{H}), 0.14(\mathrm{~s}, 3 \mathrm{H}), 0.13(\mathrm{~s}, 3 \mathrm{H}), 0.11(\mathrm{~s}, 3 \mathrm{H}), 0.06(\mathrm{~s}$, $3 \mathrm{H}) ;{ }^{13} \mathrm{C}$ NMR $\left(100 \mathrm{MHz}, \mathrm{CDCl}_{3}\right) \delta 163.26,156.78,129.71,119.78,114.47,104.59$, 103.87, 92.52, 89.12, 74.89, 69.96, 65.37, 58.50, 55.68, 29.36, 26.31, 26.10, 20.97, $18.35,18.32,17.83,-0.05,-3.35,-3.73,-4.26,-4.56$; IR (neat) 2954, 2931, 2896, 2858, $2164,1761,1513,1250,838 \mathrm{~cm}^{-1}$; HRMS [M+H] Calcd. for $\mathrm{C}_{34} \mathrm{H}_{59} \mathrm{O}_{6} \mathrm{~N}_{1}{ }^{127} \mathrm{I}_{1}{ }^{28} \mathrm{Si}_{3}$ 788.26895, Found 788.26893.

Enantiomerically pure hydroxyl beta lactam (+)-28: The glycosylated beta-lactam $\mathbf{N}$ $(2.50 \mathrm{~g}, 3.17 \mathrm{mmol})$ was dissolved in dry THF $(50 \mathrm{~mL})$, and cooled to $-78{ }^{\circ} \mathrm{C}$. MeLi $\left(2.18 \mathrm{~mL}, 1.6 \mathrm{M}\right.$ in $\mathrm{Et}_{2} \mathrm{O}$ ) was added dropwise at $-78^{\circ} \mathrm{C}$. The reaction mixure was stirred for $10 \mathrm{~min}$ at $-78{ }^{\circ} \mathrm{C}$ and quenched with aq. sat. $\mathrm{NH}_{4} \mathrm{Cl}(10 \mathrm{~mL})$. The organic layer was separated, and the aqueous layer was extracted with $\mathrm{CH}_{2} \mathrm{Cl}_{2}(3 \times 10 \mathrm{~mL})$. The combined organic layer was dried over $\mathrm{MgSO}_{4}$ and solvent removed by rotary evaporation. Chromatography (hexanes : EtOAc $=30: 1$ to $10: 1$ ) gave each separable bis-TBS protected glycal $21 \mathrm{a}$ ( $1.05 \mathrm{~g}, 92 \%$ yield) as a colorless oil, and enantiomerically pure hydroxyl beta lactam $(+)-28(0.83 \mathrm{~g}, 87 \%)$ as a white solid. MP $=116{ }^{\circ} \mathrm{C} ;[\alpha]^{23}=$ 
+193.5 (c = 1.2, MeOH); ${ }^{1} \mathrm{H}$ NMR $\left(400 \mathrm{MHz}, \mathrm{CDCl}_{3}\right) \delta 7.53(\mathrm{~d}, J=9.2 \mathrm{~Hz}, 2 \mathrm{H}), 6.82(\mathrm{~d}$, $J=9.2 \mathrm{~Hz}, 2 \mathrm{H}), 5.04(\mathrm{~s}, 1 \mathrm{H}), 3.78(\mathrm{~s}, 3 \mathrm{H}), 1.73(\mathrm{~s}, 3 \mathrm{H}), 0.17(\mathrm{~s}, 9 \mathrm{H}) ;{ }^{13} \mathrm{C}$ NMR $(100$ $\left.\mathrm{MHz}, \mathrm{CDCl}_{3}\right) \delta 166.68,156.93,129.42,119.94,114.37,104.06,92.53,83.37,59.04$, 55.56, 19.96, -0.09; IR (KBr) 3368 (brs), 2168, 1756, 1032, 941; HRMS [M+H] Calcd. for $\mathrm{C}_{16} \mathrm{H}_{22} \mathrm{O}_{3}{ }^{28} \mathrm{Si}_{1}$ 304.13640, Found 304.13562.

(-)-28: The procedure as described above for preparation of $(+)-28$ was followed with compound $\mathrm{O}(2.39 \mathrm{~g}, 3.03 \mathrm{mmol})$ and MeLi $\left(2.18 \mathrm{~mL}, 1.6 \mathrm{M}\right.$ in $\left.\mathrm{Et}_{2} \mathrm{O}\right)$ in THF (50 mL), affording each separable bis-TBS protected glycal $21 \mathrm{a}(1.05 \mathrm{~g}, 96 \%)$ as a colorless oil and (-)-28 (0.85 g, 92\%) as a white solid.

Crystal structure of (+)-28: The absolute stereochemistry of hydroxyl beta-lactam (+)28 was confirmed by single crystal X-ray analysis on crystals grown at room temperature from a mixed solution of hexanes and ethyl acetate; absolute structure parameter $0.08(3)$. The thermal ellipsoid diagram for $(+)-28$ is shown below:

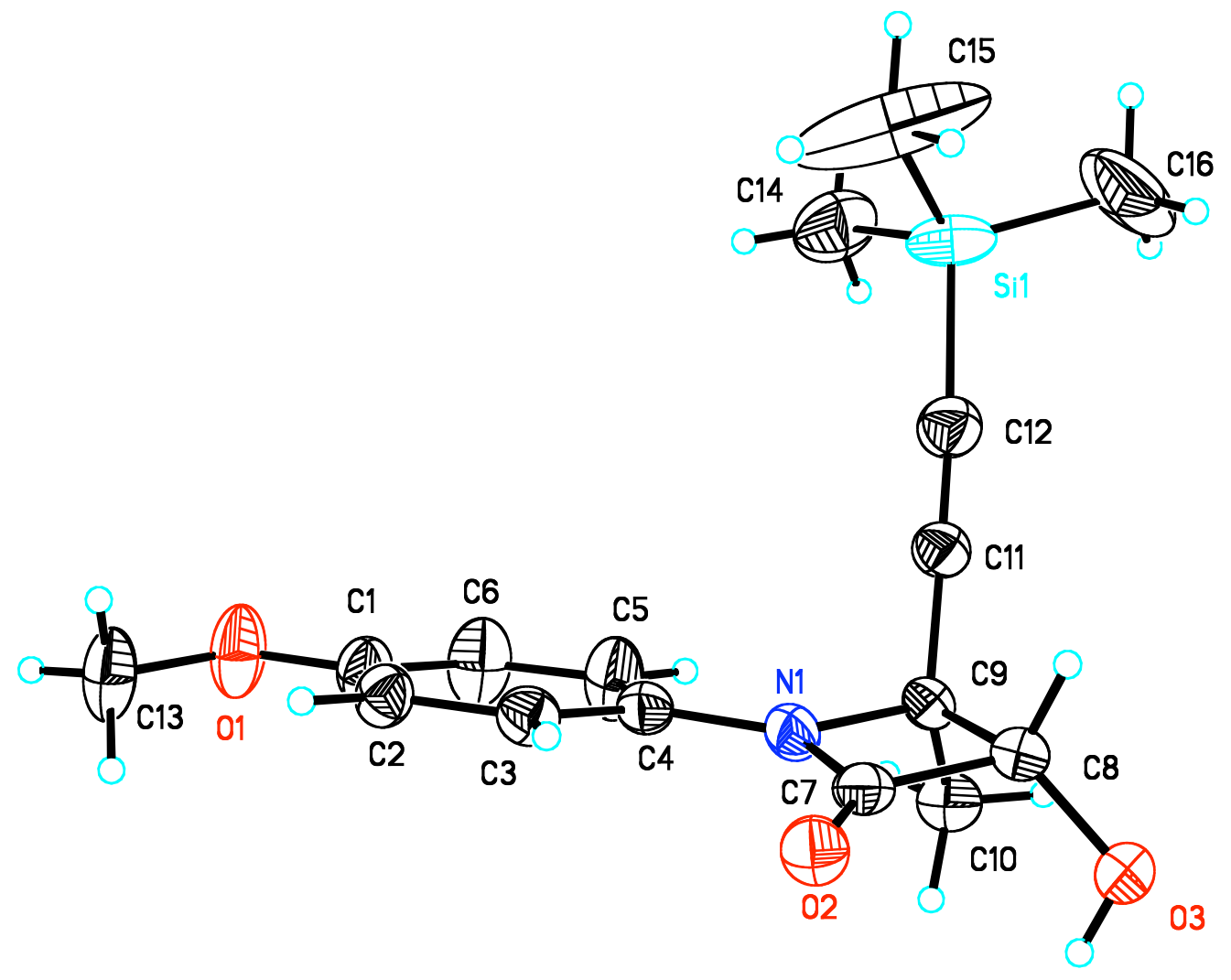


Table 1. Crystal data and structure refinement for (+)-28.

Identification code

Empirical formula

Formula weight

Temperature

Wavelength

Crystal system

Space group

Unit cell dimensions

Volume

Z

Density (calculated)

Absorption coefficient

$\mathrm{F}(000)$

Crystal size

Theta range for data collection

Index ranges

Reflections collected

Independent reflections

Completeness to theta $=65.71^{\circ}$

Max. and min. transmission

Refinement method

Data / restraints / parameters

Goodness-of-fit on $\mathrm{F}^{2}$

Final $\mathrm{R}$ indices [l>2sigma(I)]

$\mathrm{R}$ indices (all data)

Absolute structure parameter

Largest diff. peak and hole
28

$\mathrm{C}_{16} \mathrm{H}_{21} \mathrm{~N} \mathrm{O}_{3} \mathrm{Si}$

303.43

$173(2) \mathrm{K}$

$1.54178 \AA$

Monoclinic

P2(1)

$$
\begin{array}{ll}
a=10.6408(8) \AA & \alpha=90^{\circ} . \\
b=6.0649(5) \AA & \beta=101.346(4)^{\circ} . \\
c=13.6002(10) \AA & \gamma=90^{\circ} .
\end{array}
$$

$860.54(11) \AA^{3}$

2

$1.171 \mathrm{Mg} / \mathrm{m}^{3}$

$1.280 \mathrm{~mm}^{-1}$

324

$0.40 \times 0.13 \times 0.06 \mathrm{~mm}^{3}$

3.31 to $65.71^{\circ}$.

$-8<=\mathrm{h}<=12,-6<=\mathrm{k}<=6,-16<=\mathrm{l}<=15$

6072

$2403[\mathrm{R}$ (int) $=0.0200]$

$94.2 \%$

0.9272 and 0.6285

Full-matrix least-squares on $\mathrm{F}^{2}$

2403 / 1 / 196

1.016

$\mathrm{R} 1=0.0279, \mathrm{wR} 2=0.0716$

$\mathrm{R} 1=0.0293, w R 2=0.0729$

$0.08(3)$

0.142 and -0.168 e. $\AA^{-3}$ 
Table 2. Atomic coordinates $\left(\times 10^{4}\right)$ and equivalent isotropic displacement parameters $\left(\AA^{2} \times 10^{3}\right)$ for $(+)-28$. $U(e q)$ is defined as one third of the trace of the orthogonalized Uij tensor.

\begin{tabular}{lrrrr}
\hline & $\mathrm{x}$ & $\mathrm{y}$ & $\mathrm{z}$ & $\mathrm{U}(\mathrm{eq})$ \\
\hline $\mathrm{C}(1)$ & $5502(2)$ & $7544(3)$ & $1477(1)$ & $34(1)$ \\
$\mathrm{C}(2)$ & $4509(2)$ & $8835(3)$ & $963(1)$ & $31(1)$ \\
$\mathrm{C}(3)$ & $3249(2)$ & $8357(3)$ & $1027(1)$ & $30(1)$ \\
$\mathrm{C}(4)$ & $2967(2)$ & $6597(3)$ & $1602(1)$ & $28(1)$ \\
$\mathrm{C}(5)$ & $3968(2)$ & $5346(4)$ & $2129(2)$ & $45(1)$ \\
$\mathrm{C}(6)$ & $5218(2)$ & $5802(4)$ & $2056(2)$ & $48(1)$ \\
$\mathrm{C}(7)$ & $529(2)$ & $6735(3)$ & $1114(1)$ & $28(1)$ \\
$\mathrm{C}(8)$ & $-216(2)$ & $5464(3)$ & $1785(1)$ & $29(1)$ \\
$\mathrm{C}(9)$ & $1143(2)$ & $4575(3)$ & $2334(1)$ & $28(1)$ \\
$\mathrm{C}(10)$ & $1336(2)$ & $2131(4)$ & $2175(1)$ & $36(1)$ \\
$\mathrm{C}(11)$ & $1537(2)$ & $5257(3)$ & $3389(1)$ & $31(1)$ \\
$\mathrm{C}(12)$ & $1866(2)$ & $5846(4)$ & $4242(2)$ & $39(1)$ \\
$\mathrm{C}(13)$ & $7079(2)$ & $9459(4)$ & $785(2)$ & $51(1)$ \\
$\mathrm{C}(14)$ & $4095(2)$ & $5972(5)$ & $5974(2)$ & $64(1)$ \\
$\mathrm{C}(15)$ & $2338(5)$ & $9966(6)$ & $5432(3)$ & $158(3)$ \\
$\mathrm{C}(16)$ & $1368(3)$ & $5873(8)$ & $6331(2)$ & $100(2)$ \\
$\mathrm{N}(1)$ & $1684(1)$ & $6074(3)$ & $1646(1)$ & $29(1)$ \\
$\mathrm{O}(1)$ & $6774(1)$ & $7846(3)$ & $1464(1)$ & $50(1)$ \\
$\mathrm{O}(2)$ & $236(1)$ & $7902(2)$ & $371(1)$ & $33(1)$ \\
$\mathrm{O}(3)$ & $-1143(1)$ & $3965(2)$ & $1341(1)$ & $37(1)$ \\
$\mathrm{Si}(1)$ & $2425(1)$ & $6909(1)$ & $5522(1)$ & $48(1)$ \\
& & & & \\
\hline
\end{tabular}


Table 3. Bond lengths $[\AA]$ and angles $\left[{ }^{\circ}\right]$ for $(+)-28$.

\begin{tabular}{|c|c|c|c|}
\hline $\mathrm{C}(1)-\mathrm{O}(1)$ & $1.369(2)$ & $C(5)-C(4)-N(1)$ & $120.36(17)$ \\
\hline$C(1)-C(6)$ & $1.386(3)$ & $C(3)-C(4)-N(1)$ & $120.85(16)$ \\
\hline$C(1)-C(2)$ & $1.388(3)$ & $C(6)-C(5)-C(4)$ & $120.37(19)$ \\
\hline$C(2)-C(3)$ & $1.391(2)$ & $C(5)-C(6)-C(1)$ & $120.97(19)$ \\
\hline$C(3)-C(4)$ & $1.391(3)$ & $\mathrm{O}(2)-\mathrm{C}(7)-\mathrm{N}(1)$ & $131.87(16)$ \\
\hline$C(4)-C(5)$ & $1.388(3)$ & $\mathrm{O}(2)-\mathrm{C}(7)-\mathrm{C}(8)$ & $134.99(16)$ \\
\hline $\mathrm{C}(4)-\mathrm{N}(1)$ & $1.413(2)$ & $N(1)-C(7)-C(8)$ & $93.14(14)$ \\
\hline$C(5)-C(6)$ & $1.381(3)$ & $\mathrm{O}(3)-\mathrm{C}(8)-\mathrm{C}(7)$ & $118.49(14)$ \\
\hline $\mathrm{C}(7)-\mathrm{O}(2)$ & $1.223(2)$ & $\mathrm{O}(3)-\mathrm{C}(8)-\mathrm{C}(9)$ & $119.07(16)$ \\
\hline $\mathrm{C}(7)-\mathrm{N}(1)$ & $1.358(2)$ & $C(7)-C(8)-C(9)$ & $85.47(13)$ \\
\hline$C(7)-C(8)$ & $1.530(3)$ & $C(11)-C(9)-N(1)$ & $111.54(15)$ \\
\hline $\mathrm{C}(8)-\mathrm{O}(3)$ & $1.389(2)$ & $C(11)-C(9)-C(10)$ & $113.15(16)$ \\
\hline $\mathrm{C}(8)-\mathrm{C}(9)$ & $1.585(2)$ & $N(1)-C(9)-C(10)$ & $115.01(14)$ \\
\hline$C(9)-C(11)$ & $1.473(3)$ & $C(11)-C(9)-C(8)$ & $114.98(15)$ \\
\hline $\mathrm{C}(9)-\mathrm{N}(1)$ & $1.500(2)$ & $N(1)-C(9)-C(8)$ & $85.79(13)$ \\
\hline$C(9)-C(10)$ & $1.518(3)$ & $C(10)-C(9)-C(8)$ & $113.67(15)$ \\
\hline$C(11)-C(12)$ & $1.198(3)$ & $C(12)-C(11)-C(9)$ & $178.9(2)$ \\
\hline $\mathrm{C}(12)-\mathrm{Si}(1)$ & $1.842(2)$ & $C(11)-C(12)-S i(1)$ & $176.16(19)$ \\
\hline $\mathrm{C}(13)-\mathrm{O}(1)$ & $1.425(3)$ & $C(7)-N(1)-C(4)$ & $133.78(15)$ \\
\hline$C(14)-S i(1)$ & $1.852(3)$ & $\mathrm{C}(7)-\mathrm{N}(1)-\mathrm{C}(9)$ & $95.29(13)$ \\
\hline $\mathrm{C}(15)-\mathrm{Si}(1)$ & $1.860(4)$ & $C(4)-N(1)-C(9)$ & $130.92(15)$ \\
\hline \multirow[t]{2}{*}{$\mathrm{C}(16)-\mathrm{Si}(1)$} & $1.832(3)$ & $C(1)-O(1)-C(13)$ & $116.84(15)$ \\
\hline & & $C(16)-S i(1)-C(12)$ & $108.99(13)$ \\
\hline $\mathrm{O}(1)-\mathrm{C}(1)-\mathrm{C}(6)$ & $115.82(17)$ & $C(16)-S i(1)-C(14)$ & $110.98(14)$ \\
\hline $\mathrm{O}(1)-\mathrm{C}(1)-\mathrm{C}(2)$ & $125.08(17)$ & $C(12)-S i(1)-C(14)$ & $108.46(11)$ \\
\hline$C(6)-C(1)-C(2)$ & $119.10(17)$ & $C(16)-S i(1)-C(15)$ & $110.7(2)$ \\
\hline$C(1)-C(2)-C(3)$ & 119.91(18) & $C(12)-S i(1)-C(15)$ & $106.50(14)$ \\
\hline$C(2)-C(3)-C(4)$ & $120.84(17)$ & $C(14)-S i(1)-C(15)$ & $111.09(18)$ \\
\hline$C(5)-C(4)-C(3)$ & $118.78(16)$ & & \\
\hline
\end{tabular}

Symmetry transformations used to generate equivalent atoms: 
Table 4. Anisotropic displacement parameters $\left(\AA^{2} \times 10^{3}\right)$ for $(+)-28$. The anisotropic displacement factor exponent takes the form: $-2 \pi^{2}\left[h^{2} a^{*} 2 U^{11}+\ldots+2 h k a^{*} b^{*} U^{12}\right]$

\begin{tabular}{lcccccc}
\hline & $U^{11}$ & $U^{22}$ & $U^{33}$ & $U^{23}$ & $U^{13}$ & $U^{12}$ \\
\hline $\mathrm{C}(1)$ & $27(1)$ & $41(1)$ & $34(1)$ & $6(1)$ & $2(1)$ & $-4(1)$ \\
$\mathrm{C}(2)$ & $33(1)$ & $29(1)$ & $31(1)$ & $4(1)$ & $5(1)$ & $-3(1)$ \\
$\mathrm{C}(3)$ & $30(1)$ & $33(1)$ & $27(1)$ & $1(1)$ & $3(1)$ & $3(1)$ \\
$\mathrm{C}(4)$ & $28(1)$ & $35(1)$ & $21(1)$ & $-1(1)$ & $3(1)$ & $-2(1)$ \\
$\mathrm{C}(5)$ & $35(1)$ & $56(2)$ & $41(1)$ & $25(1)$ & $1(1)$ & $-3(1)$ \\
$\mathrm{C}(6)$ & $29(1)$ & $59(2)$ & $53(1)$ & $27(1)$ & $-3(1)$ & $4(1)$ \\
$\mathrm{C}(7)$ & $30(1)$ & $29(1)$ & $23(1)$ & $-4(1)$ & $3(1)$ & $0(1)$ \\
$\mathrm{C}(8)$ & $30(1)$ & $33(1)$ & $25(1)$ & $-5(1)$ & $7(1)$ & $-3(1)$ \\
$\mathrm{C}(9)$ & $31(1)$ & $33(1)$ & $21(1)$ & $0(1)$ & $8(1)$ & $-5(1)$ \\
$\mathrm{C}(10)$ & $40(1)$ & $34(1)$ & $33(1)$ & $0(1)$ & $6(1)$ & $1(1)$ \\
$\mathrm{C}(11)$ & $35(1)$ & $34(1)$ & $24(1)$ & $1(1)$ & $6(1)$ & $-3(1)$ \\
$\mathrm{C}(12)$ & $42(1)$ & $41(1)$ & $32(1)$ & $0(1)$ & $6(1)$ & $-6(1)$ \\
$\mathrm{C}(13)$ & $30(1)$ & $58(2)$ & $65(1)$ & $20(1)$ & $12(1)$ & $-6(1)$ \\
$\mathrm{C}(14)$ & $55(1)$ & $71(2)$ & $57(2)$ & $-8(1)$ & $-8(1)$ & $-4(1)$ \\
$\mathrm{C}(15)$ & $248(6)$ & $46(2)$ & $120(3)$ & $-25(2)$ & $-115(4)$ & $27(3)$ \\
$\mathrm{C}(16)$ & $78(2)$ & $192(5)$ & $34(1)$ & $-23(2)$ & $19(1)$ & $14(2)$ \\
$\mathrm{N}(1)$ & $30(1)$ & $35(1)$ & $22(1)$ & $2(1)$ & $6(1)$ & $-2(1)$ \\
$\mathrm{O}(1)$ & $25(1)$ & $61(1)$ & $62(1)$ & $25(1)$ & $5(1)$ & $-2(1)$ \\
$\mathrm{O}(2)$ & $32(1)$ & $37(1)$ & $29(1)$ & $5(1)$ & $3(1)$ & $2(1)$ \\
$\mathrm{O}(3)$ & $33(1)$ & $45(1)$ & $33(1)$ & $-6(1)$ & $7(1)$ & $-10(1)$ \\
$\mathrm{Si}(1)$ & $64(1)$ & $44(1)$ & $30(1)$ & $-11(1)$ & $-9(1)$ & $5(1)$ \\
& & & & & & \\
\hline
\end{tabular}


Table 5. Hydrogen coordinates $\left(x 10^{4}\right)$ and isotropic displacement parameters $\left(\AA^{2} x\right.$ $10^{3}$ ) for (+)-28.

\begin{tabular}{lrrrr}
\hline & $x$ & $y$ & $z$ & $U(e q)$ \\
\hline$H(2)$ & & & & \\
$H(3)$ & 4686 & 10019 & 577 & 37 \\
$H(5)$ & 2587 & 9225 & 680 & 36 \\
$H(6)$ & 3797 & 4193 & 2534 & 54 \\
$H(8)$ & 5879 & 4928 & 2400 & 58 \\
$H(10 A)$ & -569 & 6479 & 2222 & 35 \\
$H(10 B)$ & 2210 & 1743 & 2445 & 54 \\
$H(10 C)$ & 1144 & 1809 & 1470 & 54 \\
$H(13 A)$ & 777 & 1296 & 2507 & 54 \\
$H(13 B)$ & 6601 & 9163 & 123 & 76 \\
$H(13 C)$ & 7979 & 9402 & 781 & 76 \\
$H(14 A)$ & 6861 & 10898 & 994 & 76 \\
$H(14 B)$ & 4138 & 4399 & 5907 & 95 \\
$H(14 C)$ & 4368 & 6371 & 6666 & 95 \\
$H(15 A)$ & 4646 & 6659 & 5583 & 95 \\
$H(15 B)$ & 2935 & 10484 & 5038 & 237 \\
$H(15 C)$ & 2548 & 10593 & 6092 & 237 \\
$H(16 A)$ & 1486 & 10401 & 5118 & 237 \\
$H(16 B)$ & 495 & 6228 & 6039 & 150 \\
$H(16 C)$ & 1596 & 6547 & 6980 & 150 \\
$H(3 A)$ & 1460 & 4303 & 6397 & 150 \\
& -921 & 3396 & 855 & 55 \\
\hline
\end{tabular}


Table 6. Hydrogen bonds for $(+)-28\left[\AA\right.$ and $\left.{ }^{\circ}\right]$.

\begin{tabular}{lcccc}
\hline $\mathrm{D}-\mathrm{H} \ldots \mathrm{A}$ & $\mathrm{d}(\mathrm{D}-\mathrm{H})$ & $\mathrm{d}(\mathrm{H} \ldots \mathrm{A})$ & $\mathrm{d}(\mathrm{D} \ldots \mathrm{A})$ & $<(\mathrm{DHA})$ \\
\hline $\mathrm{O}(3)-\mathrm{H}(3 \mathrm{~A}) \ldots \mathrm{O}(2) \# 1$ & 0.82 & 1.97 & $2.7663(17)$ & 163.6
\end{tabular}

Symmetry transformations used to generate equivalent atoms:

$\# 1-x, y-1 / 2,-z$

\section{Preparation of glycosylated beta-lactam 29}

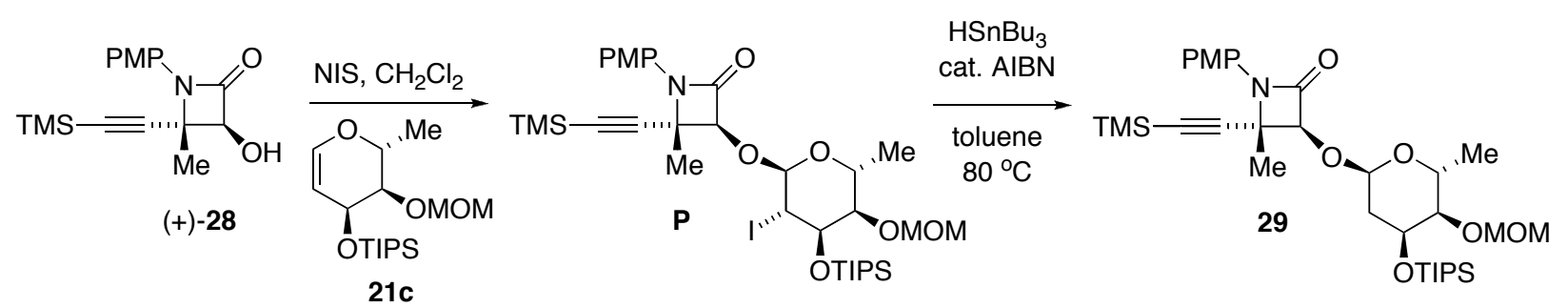

NIS mediated glycosylation: The hydroxyl beta-lactam $(+)-28(0.411 \mathrm{~g}, 1.36 \mathrm{mmol})$ and glycal 21c $(0.407 \mathrm{~g}, 1.23 \mathrm{mmol})$ were dissolved in $\mathrm{CH}_{2} \mathrm{Cl}_{2}(8 \mathrm{~mL})$, and cooled to $0{ }^{\circ} \mathrm{C}$. NIS $(0.291 \mathrm{~g}, 1.29 \mathrm{mmol})$ was added at $0{ }^{\circ} \mathrm{C}$. The mixture was slowly warmed to room temperature and then stirred overnight in the dark. The resulting solution was diluted with $\mathrm{CH}_{2} \mathrm{Cl}_{2}(5 \mathrm{~mL})$ and quenched with sat. aq. $\mathrm{Na}_{2} \mathrm{~S}_{2} \mathrm{O}_{4}(10 \mathrm{~mL})$. The organic layer was separated and the aqueous layer was extracted with $\mathrm{CH}_{2} \mathrm{Cl}_{2}(3 \times 10 \mathrm{~mL})$. The combined organic layer was dried over $\mathrm{MgSO}_{4}$ and solvent removed by rotary evaporation. Chromatography (hexanes : EtOAc $=15: 1$ ) gave iodoglycal beta-lactam $\mathbf{P}$ as a colorless oil $(0.766 \mathrm{~g}, 82 \%$ yield $) .[\alpha]^{23}=+132.9\left(c=3.45, \mathrm{CHCl}_{3}\right) ;{ }^{1} \mathrm{H}$ NMR $(400$ $\left.\mathrm{MHz}, \mathrm{CDCl}_{3}\right) \delta 7.56(\mathrm{~d}, J=9.2 \mathrm{~Hz}, 2 \mathrm{H}), 6.87(\mathrm{~d}, J=9.2 \mathrm{~Hz}, 2 \mathrm{H}), 5.23(\mathrm{~s}, 1 \mathrm{H}), 4.97(\mathrm{~s}$, $1 \mathrm{H}), 4.71(\mathrm{~d}, J=6.4 \mathrm{~Hz}, 1 \mathrm{H}), 4.68(\mathrm{dq}, J=6.4,9.6 \mathrm{~Hz}, 1 \mathrm{H}), 4.59(\mathrm{~d}, J=7.2 \mathrm{~Hz}, 1 \mathrm{H})$, $4.40(\mathrm{t}, J=2.4 \mathrm{~Hz}, 1 \mathrm{H}), 4.36(\mathrm{~d}, J=2.4 \mathrm{~Hz}, 1 \mathrm{H}), 4.04(\mathrm{dd}, J=2.0,9.6 \mathrm{~Hz}, 1 \mathrm{H}), 3.79(\mathrm{~s}$, 3H), $3.42(\mathrm{~s}, 3 \mathrm{H}), 1.64(\mathrm{~s}, 3 \mathrm{H}), 1.38(\mathrm{~d}, \mathrm{~J}=6.4 \mathrm{~Hz}, 3 \mathrm{H}), 1.06(\mathrm{~s}, 21 \mathrm{H}), 0.16(\mathrm{~s}, 9 \mathrm{H}) ;{ }^{13} \mathrm{C}$ NMR $\left(100 \mathrm{MHz}, \mathrm{CDCl}_{3}\right) \delta 162.18,156.67,129.87,119.63,114.43,104.15,102.47$, 
$95.56,92.58,86.67,74.16,72.55,64.10,57.52,56.36,55.66,29.33,20.54,18.22$ $18.13,17.86,12.76,-0.09$; IR (neat) 2942, 2252, 2162, 2057, 1950, 1871, 1760, 1513, 1373, 1247, $844 \mathrm{~cm}^{-1}$; HRMS [M+H] Calcd. for $\mathrm{C}_{33} \mathrm{H}_{55} \mathrm{O}_{7} \mathrm{~N}_{1}{ }^{127} \mathrm{I}_{1}{ }^{28} \mathrm{Si}_{2} 760.25564$, Found 760.25794 .

Deiodination: Compound $\mathbf{P}(0.550 \mathrm{~g}, 0.754 \mathrm{mmol})$ was dissolved in toluene $(25 \mathrm{~mL})$, and $\mathrm{HSnBu}_{3}(1.00 \mathrm{~mL}, 3.77 \mathrm{mmol})$ and $\operatorname{AIBN}(12.4 \mathrm{mg}, 0.075 \mathrm{mmol})$ were added. Air was removed through vacuum-argon exchange (3 times). The reaction mixture was heated to $80{ }^{\circ} \mathrm{C}$ and stirred for $5 \mathrm{~h}$. The reaction mixture was cooled to room temperature, and solvent was removed by rotary evaporation. Chromatography (hexanes : EtOAc $=40: 1$, gradient to $30: 1$ and to $20: 1$ ) gave compound 29 as a colorless oil $(0.416 \mathrm{~g}, 88 \%$ yield $) .[\alpha]^{23}{ }_{D}=+114.3\left(c=1.8, \mathrm{CHCl}_{3}\right) ;{ }^{1} \mathrm{H}$ NMR $(400 \mathrm{MHz}$, $\left.\mathrm{CDCl}_{3}\right) \delta 7.57(\mathrm{~d}, J=9.2 \mathrm{~Hz}, 2 \mathrm{H}), 6.87(\mathrm{~d}, J=9.2 \mathrm{~Hz}, 2 \mathrm{H}), 5.02(\mathrm{~s}, 1 \mathrm{H}), 4.93(\mathrm{dd}, J=$ 2.4, $4.8 \mathrm{~Hz}, 1 \mathrm{H}$ ), $4.76(\mathrm{~d}, J=7.2 \mathrm{~Hz}, 1 \mathrm{H}), 4.59$ (d, J = 7.2 Hz, 1H), 4.55 (dq, J = 6.4, 8.4 $\mathrm{Hz}, 1 \mathrm{H}$ ), 4.26 (ddd, $J=2.8,2.84 .8 \mathrm{~Hz}, 1 \mathrm{H}), 3.79$ (s, 3H), 3.40 (s, 3H), 3.37 (dd, $J=2.8$, $8.4 \mathrm{~Hz}, 1 \mathrm{H}$ ), 2.19 (ddd, $J=2.4,4.8,14.4 \mathrm{~Hz}, 1 \mathrm{H}$ ), 1.92 (ddd, $J=2.8,4.8,14.4 \mathrm{~Hz}, 1 \mathrm{H}$ ), $1.63(\mathrm{~s}, 3 \mathrm{H}), 1.34(\mathrm{~d}, J=6.4 \mathrm{~Hz}, 3 \mathrm{H}), 1.06(\mathrm{~s}, 21 \mathrm{H}), 0.15(\mathrm{~s}, 9 \mathrm{H}) ;{ }^{13} \mathrm{C} N M R(100 \mathrm{MHz}$, $\left.\mathrm{CDCl}_{3}\right) \delta 163.30,156.56,130.10,119.60,114.40,104.62,97.70,95.28,92.14,86.97$ $78.40,65.76,64.66,57.53,56.05,55.68,36.69,20.54,18.32,18.27,12.76,-0.05 ;$ IR (neat) 2941, 2866, 2162, 1763, 1512, $1248 \mathrm{~cm}^{-1}$; HRMS Calcd. [M+H] for $\mathrm{C}_{33} \mathrm{H}_{56} \mathrm{O}_{7} \mathrm{~N}_{1}{ }^{28} \mathrm{Si}_{2}$ 634.35899, Found 634.35891.

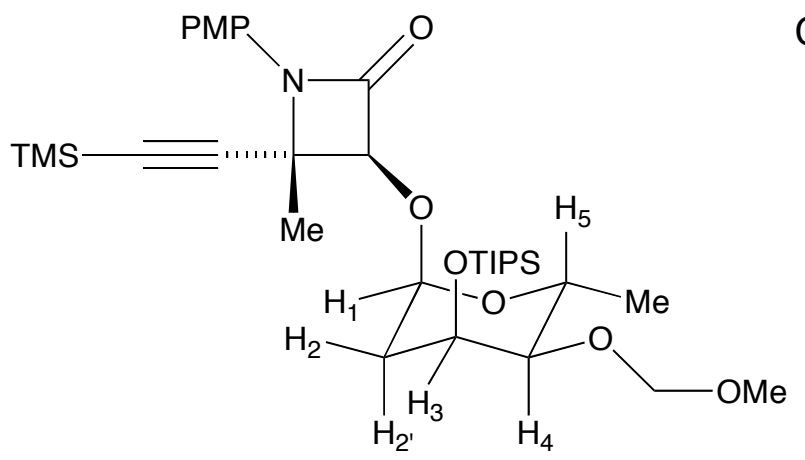

Coupling constants for 29 were consistent with the axial glycoside as shown:

$J_{1,2}=4.8 \mathrm{~Hz}$, diequatorial $\mathrm{HCCH}$ $J_{1,2^{\prime}}=2.4 \mathrm{~Hz}$, equatorial, axial $\mathrm{HCCH}$ $J_{2,3}=4.8 \mathrm{~Hz}$, diequatorial $\mathrm{HCCH}$ $J_{2,3}=2.8 \mathrm{~Hz}$, axial, equatorial $\mathrm{HCCH}$ $J_{3,4}=2.8 \mathrm{~Hz}$, equatorial, axial $\mathrm{HCCH}$ $J_{4,5}=8.4 \mathrm{~Hz}$, diaxial $\mathrm{HCCH}$ 


\section{Preparation of alkynyl ketone 30}
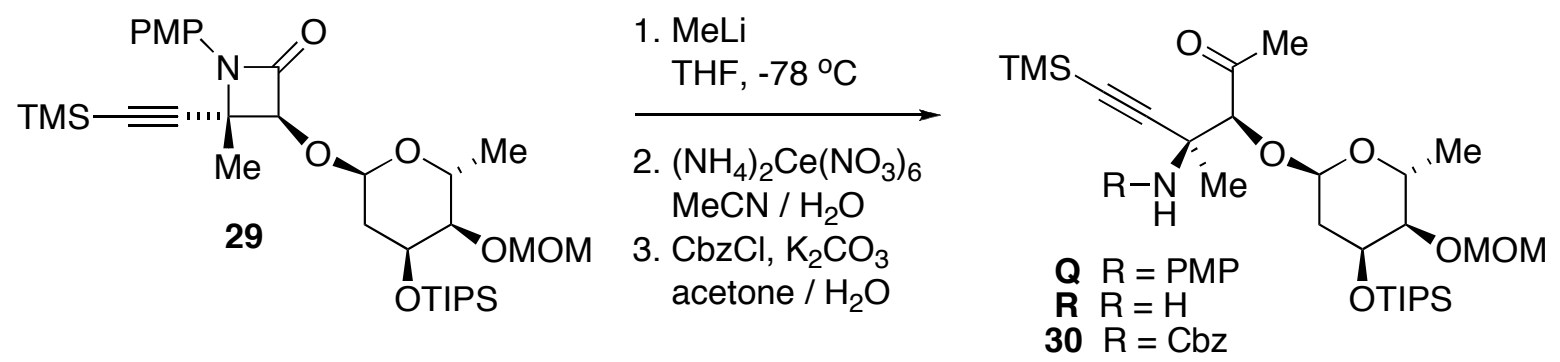

MeLi addition: Beta-lactam compound $29(0.416 \mathrm{~g}, 0.733 \mathrm{mmol})$ was dissolved in dry THF $(10 \mathrm{~mL})$ and cooled to $-78{ }^{\circ} \mathrm{C}$. MeLi $\left(0.687 \mathrm{~mL}, 1.10 \mathrm{mmol}, 1.6 \mathrm{M}\right.$ in $\left.\mathrm{Et}_{2} \mathrm{O}\right)$ was added dropwise at $-78{ }^{\circ} \mathrm{C}$. The reaction mixture was stirred for $30 \mathrm{~min}$ at $-78{ }^{\circ} \mathrm{C}$ and quenched with aq. sat. $\mathrm{NaHCO}_{3}(5 \mathrm{~mL})$. The organic layer was separated and the aqueous layer was extracted with $\mathrm{Et}_{2} \mathrm{O}(3 \times 10 \mathrm{~mL})$. The combined organic layer was dried over $\mathrm{MgSO}_{4}$ and solvent removed by rotary evaporation. Chromatography (hexanes : EtOAc $=10: 1)$ gave ketone $\mathbf{Q}$ as a colorless oil $\left(0.425 \mathrm{~g}, 90 \%\right.$ yield). $[\alpha]^{23}{ }_{D}$ $=+80.5\left(c=2.0, \mathrm{CHCl}_{3}\right) ;{ }^{1} \mathrm{H}$ NMR $\left(400 \mathrm{MHz}, \mathrm{CDCl}_{3}\right) \delta 7.03(\mathrm{~d}, J=9.2 \mathrm{~Hz}, 2 \mathrm{H}), 6.76(\mathrm{~d}$, $J=9.2 \mathrm{~Hz}, 2 \mathrm{H}), 4.94(\mathrm{dd}, J=4.0 \mathrm{~Hz}, 1 \mathrm{H}), 4.78(\mathrm{~d}, J=6.8 \mathrm{~Hz}, 1 \mathrm{H}), 4.62(\mathrm{~d}, J=6.8 \mathrm{~Hz}$, 1H), 4.24-4.17 (m, 2H), $4.00(\mathrm{~s}, 1 \mathrm{H}), 3.99(\mathrm{~d}, J=4.0 \mathrm{~Hz}, 1 \mathrm{H}), 3.76(\mathrm{~s}, 3 \mathrm{H}), 3.39(\mathrm{~s}, 3 \mathrm{H})$, $3.38(\mathrm{dd}, J=2.8,6.0 \mathrm{~Hz}, 1 \mathrm{H}), 2.35(\mathrm{~s}, 3 \mathrm{H}), 2.27$ (ddd, $J=4.0,6.4,14.0 \mathrm{~Hz}, 1 \mathrm{H}), 1.94$ (ddd, $J=4.0,14.4 \mathrm{~Hz}, 1 \mathrm{H}), 1.42(\mathrm{~s}, 3 \mathrm{H}), 1.17(\mathrm{~d}, J=6.8 \mathrm{~Hz}, 3 \mathrm{H}), 1.10(\mathrm{~s}, 21 \mathrm{H}), 0.13(\mathrm{~s}$, $9 \mathrm{H}) ;{ }^{13} \mathrm{C}$ NMR $\left(100 \mathrm{MHz}, \mathrm{CDCl}_{3}\right) \delta 209.60,154.83,138.33,122.63,114.11,107.10$, $99.91,95.90,90.53,87.80,78.19,66.61,65.99,56.42,55.98,55.72,36.65,29.17$, $24.27,18.39,18.34,17.74,12.72,-0.04$; IR (neat) 2942, 2867, 2166, 1712, 1510, 1248 , 1035, $842 \mathrm{~cm}^{-1}$; HRMS Calcd. [M+H] for $\mathrm{C}_{34} \mathrm{H}_{60} \mathrm{O}_{7} \mathrm{~N}_{1}{ }^{28} \mathrm{Si}_{2} 650.39029$, Found 650.38986 .

Removal of PMP: The ketone $\mathbf{Q}(0.425 \mathrm{~g}, 0.660 \mathrm{mmol})$ was dissolved in a mixture of $\mathrm{CH}_{3} \mathrm{CN}(12 \mathrm{~mL})$ and water $(6 \mathrm{~mL})$, and cooled to $0{ }^{\circ} \mathrm{C}$. Ceric ammonium nitrate $(0.724 \mathrm{~g}$, $1.32 \mathrm{mmol})$ dissolved in water $(6 \mathrm{~mL})$ was added dropwise at $0{ }^{\circ} \mathrm{C}$, and the reaction 
mixture was slowly warmed to room temperature and stirred for $1 \mathrm{~h}$. The resulting solution was quenched with aq. sat. $\mathrm{NaHCO}_{3}(20 \mathrm{~mL})$ and then stirred for $1 \mathrm{~h}$ at room temperature. The solution was filtered through Celite using EtOAc as eluent and the organic layer was separated. The aqueous layer was extracted with EtOAc $(3 \times 10 \mathrm{~mL})$, the combined organic layer was dried over $\mathrm{MgSO}_{4}$, and solvents were removed by rotary evaporation, to afford crude amine $\mathbf{R}$ which was used in the next step without further purification.

Cbz protection: The procedure as described above for $( \pm)-4,( \pm)-6,8$ and 14 was followed with crude amine $\mathbf{R}, \mathrm{K}_{2} \mathrm{CO}_{3}(9.1 \mathrm{mg}, 0.066 \mathrm{mmol})$ and $\mathrm{CbzCl}(0.14 \mathrm{~mL}, 0.99$ $\mathrm{mmol})$ in acetone $(6 \mathrm{~mL})$ and water $(6 \mathrm{~mL})$, to provide ketone $\mathbf{3 0}$ as a colorless oil $(0.29$ g, $65 \%$ over 2 steps) after chromatography (hexanes : EtOAc $=5: 1) .[\alpha]^{23}{ }_{D}=+87.8(c$ $\left.=3.5, \mathrm{CHCl}_{3}\right) ;{ }^{1} \mathrm{H} \mathrm{NMR}\left(400 \mathrm{MHz}, \mathrm{CDCl}_{3}\right)$ ठ 7.37-7.26 (m, 5H), $5.67(\mathrm{~s}, 1 \mathrm{H}), 5.07(\mathrm{~s}, 2 \mathrm{H})$, $4.81(\mathrm{dd}, J=3.2,5.2 \mathrm{~Hz}, 1 \mathrm{H}), 4.74(\mathrm{~d}, J=6.8 \mathrm{~Hz}, 1 \mathrm{H}), 4.58(\mathrm{~d}, J=6.8 \mathrm{~Hz}, 1 \mathrm{H}), 4.12$ (ddd, $J=3.2,6.0 \mathrm{~Hz}, 1 \mathrm{H}$ ), $4.11-4.07(\mathrm{~m}, 2 \mathrm{H}), 3.37(\mathrm{~s}, 3 \mathrm{H}), 3.31$ (dd, $J=2.8,8.0 \mathrm{~Hz}$, 1H), 2.35 (s, 3H), 2.16 (ddd, $J=2.8,5.6,14.0 \mathrm{~Hz}, 1 \mathrm{H}$ ), 1.85 (ddd, $J=3.6,5.2,14.0 \mathrm{~Hz}$, 1H), $1.65(\mathrm{~s}, 3 \mathrm{H}), 1.12(\mathrm{~d}, J=6.8 \mathrm{~Hz}, 3 \mathrm{H}), 1.06(\mathrm{~s}, 21 \mathrm{H}), 0.13(\mathrm{~s}, 9 \mathrm{H}) ;{ }^{13} \mathrm{C}$ NMR $(100$ $\left.\mathrm{MHz}, \mathrm{CDCl}_{3}\right) \delta 208.41,154.58,136.71,128.60,128.32,128.16,109.96,99.91,95.60$ $88.94,86.10,78.11,66.62,65.79,65.70,55.97,52.80,36.54,30.24,24.29,18.29$, 18.23, 17.70, 12.63, -0.03; IR (neat) 2943, 2867, 2173, 1726, 1504, 1248, 1036, 843 $\mathrm{cm}^{-1}$; HRMS [M+Na] Calcd. for $\mathrm{C}_{35} \mathrm{H}_{59} \mathrm{O}_{8} \mathrm{~N}_{1}{ }^{23} \mathrm{Na}_{1}{ }^{28} \mathrm{Si}_{2} 700.36715$, Found 700.36652. 


\section{Preparation of alkynyl alcohol 31}

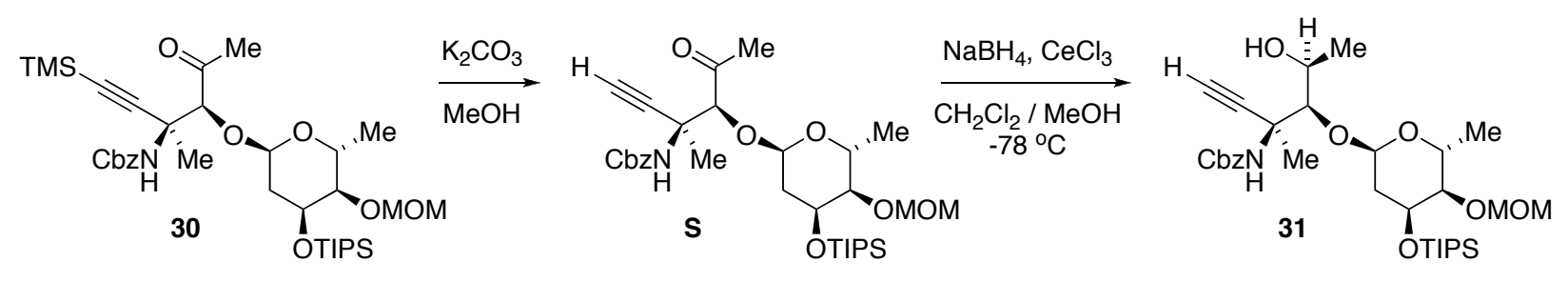

Desilylation: The alkynylsilane compound $30(0.15 \mathrm{~g}, 0.22 \mathrm{mmol})$ was dissolved in $\mathrm{MeOH}(8 \mathrm{~mL}), \mathrm{K}_{2} \mathrm{CO}_{3}(6.0 \mathrm{mg}, 0.044 \mathrm{mmol})$ was added, and the reaction mixture was stirred overnight at room temperature. The reaction was quenched with aq. sat. $\mathrm{NH}_{4} \mathrm{Cl}$ (5 mL) and diluted with $\mathrm{CH}_{2} \mathrm{Cl}_{2}(10 \mathrm{~mL})$. The organic layer was separated and the aqueous layer was extracted with $\mathrm{CH}_{2} \mathrm{Cl}_{2}(3 \times 5 \mathrm{~mL})$. The combined organic layer was dried over $\mathrm{MgSO}_{4}$ and solvent was removed by rotary evaporation. Chromatography (hexanes : EtOAc $=10: 1)$ gave terminal alkyne $\mathbf{S}$ as a colorless oil $(0.13 \mathrm{~g}, 95 \%$ yield). $[\alpha]^{23}{ }_{\mathrm{D}}=+86.5\left(c=2.0, \mathrm{CHCl}_{3}\right) ;{ }^{1} \mathrm{H}$ NMR $\left(400 \mathrm{MHz}, \mathrm{CDCl}_{3}\right) \delta$ 7.36-7.27 (m, 5H), $5.75(\mathrm{~s}$, 1H), 5.16-5.03 (m, 2H), $4.85(\mathrm{dd}, J=3.2,5.2 \mathrm{~Hz}, 1 \mathrm{H}), 4.75(\mathrm{~d}, J=7.2 \mathrm{~Hz}, 1 \mathrm{H}), 4.58(\mathrm{~d}$, $J=7.2 \mathrm{~Hz}, 1 \mathrm{H}), 4.17(\mathrm{ddd}, J=3.2,6.0 \mathrm{~Hz}, 1 \mathrm{H}), 4.14-4.07(\mathrm{~m}, 2 \mathrm{H}), 3.37(\mathrm{~s}, 3 \mathrm{H}), 3.20$ (dd, $J=3.5,8.0 \mathrm{~Hz}, 1 \mathrm{H}$ ), $2.44(\mathrm{~s}, 1 \mathrm{H}), 2.36(\mathrm{~s}, 3 \mathrm{H}), 2.18$ (ddd, $J=3.6,6.0,14.4 \mathrm{~Hz}, 1 \mathrm{H}$ ), $1.88(\mathrm{ddd}, J=3.6,4.8,14.4 \mathrm{~Hz}, 1 \mathrm{H}), 1.66(\mathrm{~s}, 3 \mathrm{H}), 1.13(\mathrm{~d}, J=6.4 \mathrm{~Hz}, 3 \mathrm{H}), 1.05$ (s, $21 \mathrm{H}) ;{ }^{13} \mathrm{C}$ NMR $\left(100 \mathrm{MHz}, \mathrm{CDCl}_{3}\right) \delta 208.07,154.76,136.54,128.63,128.43,128.24$, $100.09,95.61,85.70,83.39,78.03,72.68,66.77,65.86,65.73,56.00,52.19,36.49$, $30.50,24.15,18.38,18.29,18.22,17.79,12.63$; IR (neat) 2943, 2867, 2173, 1726, 1504 , 1248, 1036, $843 \mathrm{~cm}^{-1}$; HRMS [M+Na] Calcd. for $\mathrm{C}_{32} \mathrm{H}_{51} \mathrm{O}_{8} \mathrm{~N}_{1}{ }^{23} \mathrm{Na}_{1}{ }^{28} \mathrm{Si}_{1}$ 628.32762, Found 628.32721 . 
Diastereoselective reduction of ketone: Ketone $\mathbf{S}(0.13 \mathrm{~g}, 0.21 \mathrm{mmol})$ was dissolved in a mixture of $\mathrm{CH}_{2} \mathrm{Cl}_{2}(4 \mathrm{~mL})$ and $\mathrm{MeOH}(2 \mathrm{~mL})$, and $\mathrm{CeCl}_{3} \cdot 7 \mathrm{H}_{2} \mathrm{O}(0.16 \mathrm{~g}, 0.42 \mathrm{mmol})$ was added. The reaction mixture was stirred for $20 \mathrm{~min}$ at room temperature and then cooled to $-78{ }^{\circ} \mathrm{C} . \mathrm{NaBH}_{4}(8.0 \mathrm{mg}, 0.21 \mathrm{mmol})$ was added to the reaction mixture and then stirred for $1 \mathrm{~h}$ at $-78{ }^{\circ} \mathrm{C}$. An additional portion of $\mathrm{NaBH}_{4}(8.0 \mathrm{mg}, 0.21 \mathrm{mmol})$ was added and then stirred for $1 \mathrm{hr}$ at $-78{ }^{\circ} \mathrm{C}$. The reaction mixture was quenched with aq. sat. $\mathrm{NaHCO}_{3}(4 \mathrm{~mL})$ and then diluted with $\mathrm{CH}_{2} \mathrm{Cl}_{2}(5 \mathrm{~mL})$. The organic layer was separated and the aqueous layer was extracted with $\mathrm{CH}_{2} \mathrm{Cl}_{2}(3 \times 5 \mathrm{~mL})$. The combined organic layer was dried over $\mathrm{MgSO}_{4}$ and solvent was removed by rotary evaporation. Chromatography (hexanes : EtOAc $=10: 1$ ) gave alkynyl alcohol 31 as a colorless oil $(0.11 \mathrm{~g}, 89 \%$ yield $) .[\alpha]^{23} \mathrm{D}=+93.2\left(c=1.2, \mathrm{CHCl}_{3}\right) ;{ }^{1} \mathrm{H}$ NMR $\left(400 \mathrm{MHz}, \mathrm{CDCl}_{3}\right) \delta 7.35-$ $7.27(\mathrm{~m}, 5 \mathrm{H}), 6.12(\mathrm{~s}, 1 \mathrm{H}), 5.13-5.01(\mathrm{~m}, 3 \mathrm{H}), 4.79(\mathrm{~d}, J=6.8 \mathrm{~Hz}, 1 \mathrm{H}), 4.60(\mathrm{~d}, J=7.2$ $\mathrm{Hz}, 1 \mathrm{H}), 4.26(\mathrm{ddd}, J=6.8,14.0 \mathrm{~Hz}, 1 \mathrm{H}), 4.23-4.12(\mathrm{~m}, 2 \mathrm{H}), 3.66(\mathrm{~d}, J=1.2 \mathrm{~Hz}, 1 \mathrm{H})$, 3.40 (s, 3H), 3.38 (dd, J = 3.6, 7.6 Hz, 1H), 2.84 (d, $J=10.4 \mathrm{~Hz}, 1 \mathrm{H}), 2.37$ (s, 1H), 2.23 (ddd, $J=3.6,6.0,14.0 \mathrm{~Hz}, 1 \mathrm{H}$ ), 1.94 (ddd, $J=3.6,7.6,14.0 \mathrm{~Hz}, 1 \mathrm{H}$ ), $1.64(\mathrm{~s}, 3 \mathrm{H}), 1.28$ $(\mathrm{d}, J=6.4 \mathrm{~Hz}, 3 \mathrm{H}), 1.26(\mathrm{~d}, J=6.4 \mathrm{~Hz}, 3 \mathrm{H}), 1.06(\mathrm{~s}, 21 \mathrm{H}) ;{ }^{13} \mathrm{C} \mathrm{NMR}\left(100 \mathrm{MHz}, \mathrm{CDCl}_{3}\right)$ $\delta 155.09,136.69,128.61,128.35,128.20,99.25,95.70,84.87,77.75,71.52,66.63$, $66.21,65.99,65.95,56.13,55.10,36.78,23.73,22.85,18.31,18.19,17.82,12.69 ;$ IR (neat) 2943, 1726, 1514, 1249, $1035 \mathrm{~cm}^{-1}$; HRMS [M+Na] Calcd. for $\mathrm{C}_{32} \mathrm{H}_{53} \mathrm{O}_{8} \mathrm{~N}_{1}{ }^{23} \mathrm{Na}_{1}{ }^{28} \mathrm{Si}_{1} 630.34327$, Found 630.34292. 


\section{Synthesis of disaccharide glycal 32 via alkynyl alcohol cycloisomerization}
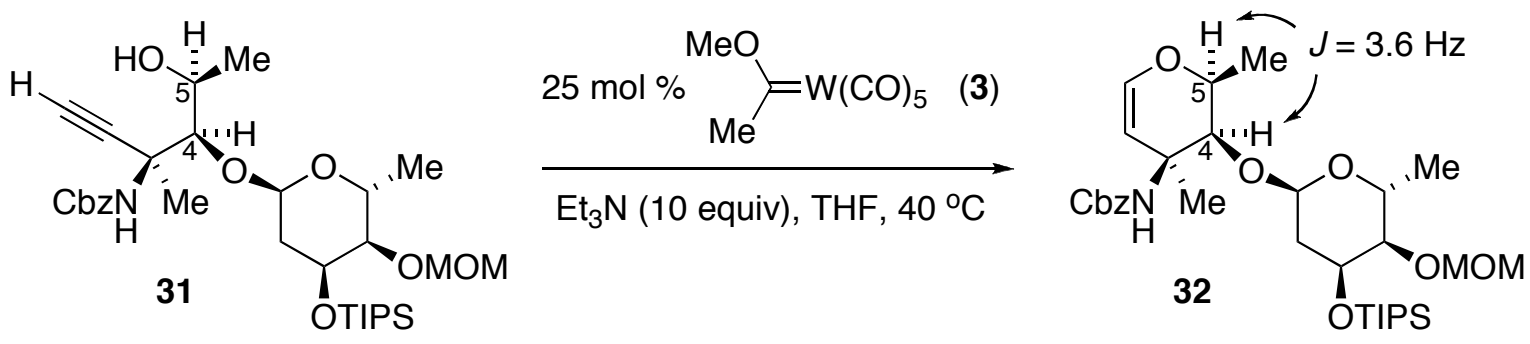

Disaccharide glycal 32: Following the general procedure for alkynyl alcohol cycloisomerization, substrate $31(0.114 \mathrm{~g}, 0.188 \mathrm{mmol})$ afforded disaccharide glycal 32 as a colorless oil (95 mg, $83 \%$ yield). At this stage, the relative stereochemistry between $\mathrm{C} 4$ and $\mathrm{C} 5$, set in the previous ketone reduction step to provide $\mathbf{3 1}$, could be determined, based on the relatively small coupling constant of $3.6 \mathrm{~Hz} .[\alpha]^{23}{ }_{\mathrm{D}}=+78.7(\mathrm{c}$ = 3.3, $\left.\mathrm{CHCl}_{3}\right) ;{ }^{1} \mathrm{H}$ NMR $\left(400 \mathrm{MHz}, \mathrm{CDCl}_{3}\right)$ ס 7.34-7.28 (m, 5H), $6.18(\mathrm{~d}, J=6.4 \mathrm{~Hz}, 1 \mathrm{H})$, $5.35(\mathrm{~s}, 1 \mathrm{H}), 5.08-5.01(\mathrm{~m}, 3 \mathrm{H}), 4.88(\mathrm{dd}, J=3.2,5.2 \mathrm{~Hz}, 1 \mathrm{H}), 4.76(\mathrm{~d}, J=7.2 \mathrm{~Hz}, 1 \mathrm{H})$, $4.59(\mathrm{~d}, J=7.2 \mathrm{~Hz}, 1 \mathrm{H}), 4.31-4.21(\mathrm{~m}, 2 \mathrm{H}), 4.15(\mathrm{ddd}, J=3.2,5.2 \mathrm{~Hz}, 1 \mathrm{H}), 3.71(\mathrm{~d}, J=$ $3.6 \mathrm{~Hz}, 1 \mathrm{H}), 3.39$ (s, 3H), 3.34 (dd, $J=2.4,3.6 \mathrm{~Hz}, 1 \mathrm{H}), 2.14$ (ddd, $J=3.2,5.6,14.0 \mathrm{~Hz}$, 1H), 1.83 (ddd, $J=3.6,14.0 \mathrm{~Hz}, 1 \mathrm{H}), 1.48(\mathrm{~s}, 3 \mathrm{H}), 1.38(\mathrm{~d}, J=6.8 \mathrm{~Hz}, 1 \mathrm{H}), 1.23(\mathrm{~d}, J=$ $6.8 \mathrm{~Hz}, 1 \mathrm{H}), 1.04(\mathrm{~s}, 21 \mathrm{H}) ;{ }^{13} \mathrm{C}$ NMR $\left(100 \mathrm{MHz}, \mathrm{CDCl}_{3}\right) \delta$ 155.14, 141.69, 136.91, $128.63,128.28,128.15,104.14,99.81,95.67,80.44,78.23,71.67,66.37,65.95,65.38$, 56.02, 51.24, 36.84, 27.03, 18.32, 18.22, 15.20, 2.65; IR (neat) 2940, 2867, 1726, 1495, $1035 \mathrm{~cm}^{-1}$; HRMS [M+Na] Calcd. for $\mathrm{C}_{32} \mathrm{H}_{53} \mathrm{O}_{8} \mathrm{~N}_{1}{ }^{23} \mathrm{Na}_{1}{ }^{28} \mathrm{Si}_{1}$ 630.34327, Found 630.34292 . 


\section{Synthesis of disaccharide glycal 33}
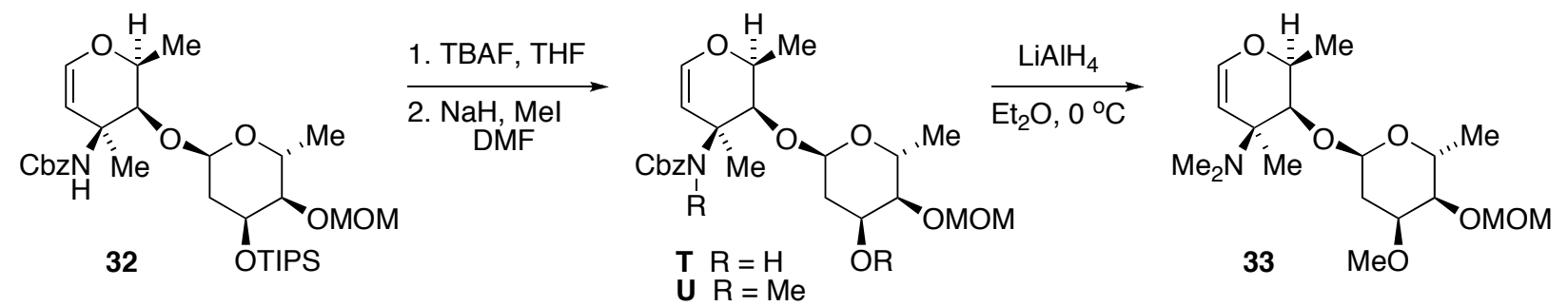

Desilylation: Silyl ether 32 (60 mg, $0.099 \mathrm{mmol}$ ) was dissolved in dry THF (3 mL), and $\operatorname{TBAF}(0.2 \mathrm{~mL}, 1 \mathrm{M}$ in THF, $0.2 \mathrm{mmol})$ was added. The reaction mixture was stirred for $30 \mathrm{~min}$ at room temperature. The reaction was diluted with EtOAc (2 mL) and quenched with water $(3 \mathrm{~mL})$. The organic layer was separated and the aqueous layer was extracted with EtOAc $(3 \times 5 \mathrm{~mL})$ and washed with brine. The combined organic layer was dried over $\mathrm{MgSO}_{4}$ and solvent removed by rotary evaporation. The crude material T was used for the next step without further purification.

N,O-Dimethylation: The crude amino alcohol T was dissolved in dry DMF (3 mL), and cooled to $0{ }^{\circ} \mathrm{C}$. Sodium hydride $(16 \mathrm{mg}, 0.40 \mathrm{mmol}$ ) was added, and the mixture was slowly warmed to room temperature and stirred for $20 \mathrm{~min}$. lodomethane ( $31 \mu \mathrm{L}, 0.50$ $\mathrm{mmol}$ ) was added to the reaction mixture and then stirred for $1 \mathrm{~h}$ at room temperature. The reaction mixture was carefully quenched with water $(1 \mathrm{~mL})$ and then diluted with EtOAc. The organic layer was separated and the aqueous layer was extracted with EtOAc $(3 \times 5 \mathrm{~mL})$. The combined organic layer was dried over $\mathrm{MgSO}_{4}$ and solvent removed by rotary evaporation. Chromatography (hexanes : EtOAc $=5: 1$ ) gave $N$ - and O-methylated product $\mathbf{U}$ as a colorless oil $(42 \mathrm{mg}, 85 \%$ over 2 steps $) .[\alpha]^{23}{ }_{D}=+6.4(c=$ 1.4, $\left.\mathrm{CHCl}_{3}\right) ;{ }^{1} \mathrm{H}$ NMR $\left(400 \mathrm{MHz}, \mathrm{CDCl}_{3}\right) \delta$ 7.29-7.18 (m, 5H), $6.19(\mathrm{~d}, J=6.4 \mathrm{~Hz}, 1 \mathrm{H})$, $5.09(\mathrm{~d}, J=12.0 \mathrm{~Hz}, 1 \mathrm{H}), 4.89(\mathrm{~d}, J=12.4 \mathrm{~Hz}, 1 \mathrm{H}), 4.63(\mathrm{~d}, J=6.8 \mathrm{~Hz}, 1 \mathrm{H}), 4.62(\mathrm{dd}, J$ $=2.4,6.4 \mathrm{~Hz}, 1 \mathrm{H}), 4.56(\mathrm{dd}, J=3.6,4.4 \mathrm{~Hz}, 1 \mathrm{H}), 4.54(\mathrm{~d}, J=6.8 \mathrm{~Hz}, 1 \mathrm{H}), 4.18$ (ddd, $J=$ 
2.8, $14.0 \mathrm{~Hz}, 1 \mathrm{H}$ ), 4.03 (brs, 1H), 3.98 (d, J = 2.4 Hz, 1H), 3.39 (brs, 1H), 3.27 (s, 3H), $3.22(\mathrm{dd}, J=3.2,7.6 \mathrm{~Hz}, 1 \mathrm{H}), 3.20(\mathrm{~s}, 3 \mathrm{H}), 2.93(\mathrm{~s},(3 \mathrm{H}), 1.89(\mathrm{~d}, J=12.0 \mathrm{~Hz}, 1 \mathrm{H}), 1.53$ (s, 3H), 1.40-1.29 (m, 1H), $1.23(\mathrm{~d}, J=6.8 \mathrm{~Hz}, 1 \mathrm{H}), 1.06(\mathrm{~d}, J=6.4 \mathrm{~Hz}, 1 \mathrm{H}) ;{ }^{13} \mathrm{C}$ NMR $\left(100 \mathrm{MHz}, \mathrm{CDCl}_{3}\right) \delta 142.96,128.22,128.42,127.98,104.78,97.45,95.53,77.84,77.31$, $73.34,71.78,66.70,65.23,58.81,55.91,55.38,31.10,17.61$; IR (neat) 2932, 1964 , 1345, 1105, $1040 \mathrm{~cm}^{-1}$; HRMS [M+Na] Calcd. for $\mathrm{C}_{25} \mathrm{H}_{37} \mathrm{O}_{8} \mathrm{~N}_{1}{ }^{23} \mathrm{Na}_{1} 502.24114$, Found 502.24075 .

$\mathrm{LiAlH}_{4}$ reduction: Compound $\mathrm{U}$ obtained above $(42 \mathrm{mg}, 0.084 \mathrm{mmol})$ was dissolved in dry $\mathrm{Et}_{2} \mathrm{O}(3 \mathrm{~mL})$ and cooled to $0{ }^{\circ} \mathrm{C}$. $\mathrm{LiAlH}_{4}\left(0.25 \mathrm{~mL}, 1 \mathrm{M}\right.$ solution in $\left.\mathrm{Et}_{2} \mathrm{O}\right)$ was added dropwise at $0{ }^{\circ} \mathrm{C}$. The reaction mixture was slowly warmed to room temperature and stirred for $5 \mathrm{~h}$. The reaction mixture was diluted with $\mathrm{Et}_{2} \mathrm{O}(2 \mathrm{~mL})$, quenched with water $(0.05 \mathrm{~mL})$ and $3 \mathrm{M} \mathrm{NaOH}(0.1 \mathrm{~mL})$, and then stirred for additional $1 \mathrm{~h}$. The solution was then dried over $\mathrm{MgSO}_{4}$ and solvent removed by rotary evaporation. Chromatography (hexanes : EtOAc $=5: 1$, gradient to $2: 1$ and to $1: 1$ ) gave the disaccharide glycal 33 as a yellowish oil $(28 \mathrm{mg}, 94 \%$ yield $) .[\alpha]^{23}{ }_{\mathrm{D}}=+83.6\left(c=1.2, \mathrm{CHCl}_{3}\right) ;{ }^{1} \mathrm{H}$ NMR $(400$ $\left.\mathrm{MHz}, \mathrm{CDCl}_{3}\right) \delta 6.27(\mathrm{~d}, J=6.4 \mathrm{~Hz}, 1 \mathrm{H}), 5.23(\mathrm{dd}, J=1.6,4.8 \mathrm{~Hz}, 1 \mathrm{H}), 4.78(\mathrm{~d}, J=6.8$ $\mathrm{Hz}, 1 \mathrm{H}), 4.68-4.66(\mathrm{~m}, 2 \mathrm{H}), 4.30(\mathrm{dq}, J=6.6,8.8 \mathrm{~Hz}, 1 \mathrm{H}), 4.16(\mathrm{dq}, J=2.4,6.4 \mathrm{~Hz}, 1 \mathrm{H})$, $3.72(\mathrm{dd}, J=2.8,7.2 \mathrm{~Hz}, 1 \mathrm{H}), 3.58(\mathrm{dd}, J=2.4 \mathrm{~Hz}, 1 \mathrm{H}), 3.41(\mathrm{~s}, 3 \mathrm{H}), 3.40(\mathrm{~s}, 3 \mathrm{H}), 3.34$ (dd, $J=2.8,8.8 \mathrm{~Hz}, 1 \mathrm{H}$ ), 2.37 (ddd, $J=2.0,4.4,14.8 \mathrm{~Hz}, 1 \mathrm{H}$ ), 2.30 (s, 6H), 1.70 (ddd, $J$ $=2.8,4.8,14.8 \mathrm{~Hz}, 1 \mathrm{H}), 1.41(\mathrm{~d}, J=6.0 \mathrm{~Hz}, 3 \mathrm{H}), 1.22(\mathrm{~d}, J=6.8 \mathrm{~Hz}, 1 \mathrm{H}), 1.11(\mathrm{~s}, 3 \mathrm{H})$; ${ }^{13} \mathrm{C}$ NMR $\left(100 \mathrm{MHz}, \mathrm{CDCl}_{3}\right) \delta 142.73,104.57,97.30,95.75,78.86,78.60,73.56,73.24$, 64.22, 56.21, 55.73, 40.62, 31.13, 22.97, 18.14, 17.27; IR (neat) 2929, 1111, 1042, 985 $\mathrm{cm}^{-1} ;[\mathrm{M}+\mathrm{H}]$ Calcd. for $\mathrm{C}_{18} \mathrm{H}_{34} \mathrm{O}_{6} \mathrm{~N}_{1} 360.23806$, Found 360.23788 . 